\title{
Path Loss Channel Model for Inland River Radio Propagation at $1.4 \mathrm{GHz}$
}

\author{
Junyi Yu, ${ }^{1}$ Wei Chen, ${ }^{1}$ Kun Yang, ${ }^{2}$ Changzhen $\mathrm{Li}^{1}{ }^{1}$ Fang Li, ${ }^{1}$ and Yishui Shui ${ }^{3}$ \\ ${ }^{1}$ School of Automation, Wuhan University of Technology, Wuhan, Hubei 430070, China \\ ${ }^{2}$ Super Radio AS, Oslo, Norway \\ ${ }^{3}$ School of Information Engineering, Wuhan University of Technology, Hubei 430070, China \\ Correspondence should be addressed to Kun Yang; kunyangntnu@gmail.com
}

Received 3 October 2016; Accepted 14 December 2016; Published 16 January 2017

Academic Editor: Claudio Gennarelli

Copyright (C) 2017 Junyi Yu et al. This is an open access article distributed under the Creative Commons Attribution License, which permits unrestricted use, distribution, and reproduction in any medium, provided the original work is properly cited.

\begin{abstract}
In this paper, a propagation path loss model for inland river is proposed by three improvements compared with the Round Earth Loss (REL) model for open-sea environment. Specifically, parameters optimization uses Okumura-Hata model in dB scale to replace the equation transformed from the free space loss in REL model; secondly, diffraction loss caused by the obstacles (e.g., large buildings, bridges, or some other facilities near the river bank) is also taken into account; mixed-path methodology as another improvement is used for Inland River (IR) model because the actual propagation environment between transmitter (TX) antenna and receiver (RX) antenna contains both land part and water part. The paper presents a set of $1.4 \mathrm{GHz}$ measurements conducted along the Yangtze River in Wuhan. According to the comparison between path loss models and experimental results, IR model shows a good matching degree. After that, Root Mean Square Error (RMSE), Grey Relation Grade and Mean Absolute Percentage Error (GRG-MAPE), Pearson Correlation Coefficient, and Mean Absolute Percentage Error (PCC-MAPE) are employed to implement quantitative analysis. The results prove that IR model with consideration of mixed path and deterministic information is more accurate than other classic empirical propagation models for these scenarios.
\end{abstract}

\section{Introduction}

As the third longest river of the world, Yangtze River plays an important role in Chinese water transportation. Our investigation in Changjiang Maritime Safety Administration showed that the whole basin navigation mileage of Yangtze River was up to 80000 kilometers (including main-stream and tributaries), accounting for about 65 percent of China inland waterways navigation mileage. In 2015, large-scale ports along the trunk line of the Yangtze River completed cargo throughput 2.118 billion tons. Thus, it is essential to provide high data rate and reliable communication service between ship to ship and ship to shore to ensure the safety of navigation. However, the performance of present broadband communication systems have not been optimized for the maritime applications due to lack of a comprehensive Inland River channel model, which is indispensable to the system design and optimization. To solve the above challenge, we propose a dedicated IR model in this article.
The inland river terrain profile contains both land and water regions. For the land area, various measurement campaigns have been performed to develop the radio channel models (e.g., Okumura-Hata empirical model [1-4]). The COST-231 Hata model [5] extending frequency adaption of Okumura-Hata is valid in frequency range from 1.5 to 2.0 GHz. However, some of key propagation phenomena have not been considered by either of them, including scattering, divergence, and shadowing. REL model derived from TwoRay theory is proposed in [6] for the open-sea scenario, which has taken effective reflection and shadowing effect due to the roughness of sea surface as well as divergence and diffraction phenomena caused by earth curvature into account. But unlike the case of open-sea, the influence of the obstacles near the inland river (e.g., diffraction loss caused by some large bridges) and mixed-path effect cannot be neglected. Paper [7] provided a methodology of field strength estimation in distance ranged from $1 \mathrm{~km}$ to $1000 \mathrm{~km}$, and frequency ranged from $30 \mathrm{MHz}$ to $3000 \mathrm{MHz}$. It is applied for land-path, 


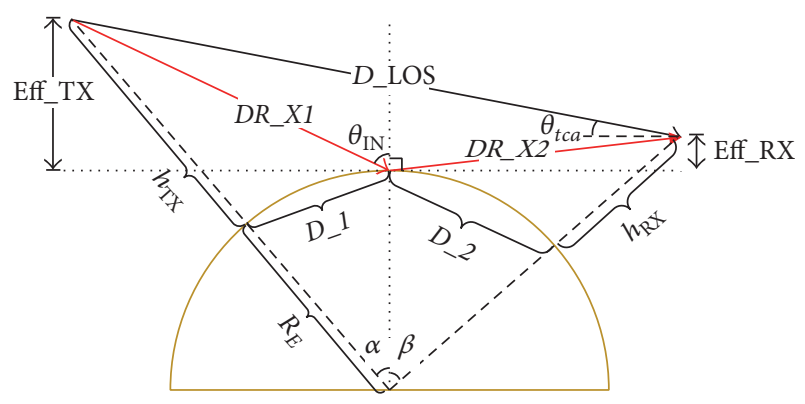

FIGURE 1: Round Earth Loss model.

sea-path, and mixed-path scenarios. However, propagation effects including scattering, divergence, and earth curvature still have not been included either. Consequently, the IR model will take these effect factors into account and make some quantitative analysis to improve the effectiveness and accuracy.

The rest of paper is organized as below: In Section 2, our new IR model is proposed based on REL model. Three typical measurement campaigns are introduced in detail in Section 3. Section 4 mainly includes analysis on finite dimensions diffraction loss as well as study on other key propagation phenomena such as effective reflection, shadowing effect on the reflected ray, divergence, and earth curvature effect. Afterward, the parameters estimation of IR model and two simulation verifications are given in Section 5. In Section 6, path loss models' effectiveness and adaptability are evaluated by using RMSE, GRG-MAPE, and PCC-MAPE algorithms, respectively. Finally, conclusions are drawn in Section 7.

\section{Path Loss Model for Inland River}

2.1. Round Earth Loss Model. Plain Earth Loss (PEL) model based on Two-Ray propagation path is commonly used for cellular communication [1]. However, the earth cannot be regarded as a "plane" when the TX-RX distance exceeds several kilometers. By accounting for scattering, divergence, shadowing, and earth curvature effects, REL model is proposed for open-sea environment [6] and its geometrical model is given in Figure 1.

The $h_{\mathrm{TX}}$ and $h_{\mathrm{RX}}$ are corresponding to the height of TX and RX antennas, respectively. $R_{E}$ which is assumed as an approximate value $6731 \mathrm{~km}$, denotes earth radius. Propagation path loss $\mathrm{PL}_{e}$ is given by

$$
\mathrm{PL}_{e}=\left(\frac{\lambda}{4 \pi D}\right)^{2} \cdot\left|1+R \cdot e^{j(2 \pi / \lambda) D_{\mathrm{diff}}}\right|^{2}
$$

where $(2 \pi / \lambda) \cdot D_{\text {diff }}$ represents the phase difference between reflected wave and line of sight (LOS) wave. $D$ is equal to D_LOS which denotes the length of LOS path. $R$ is the specular reflection coefficient $[2,8]$

$$
D_{\text {diff }}=D R \_X 1+D R \_X 2-D \text {, }
$$

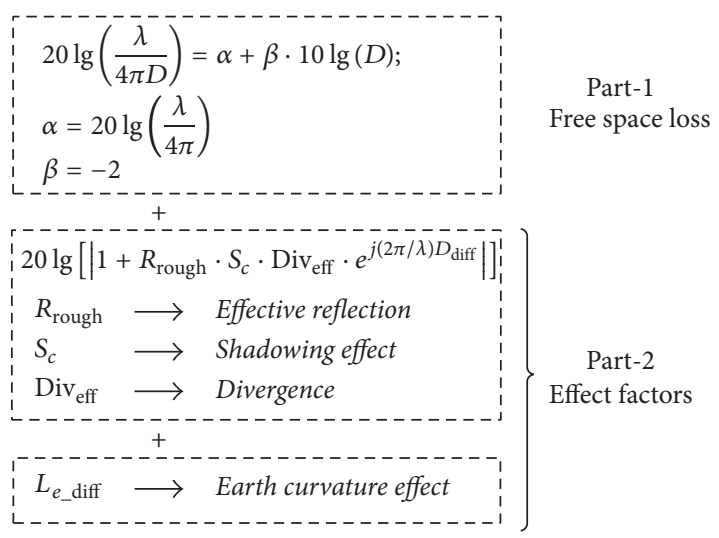

FIGURE 2: The structure of REL model.

where $D$ is calculated by

$$
\begin{aligned}
D^{2}= & \left(R_{E}+h_{\mathrm{TX}}\right)^{2}+\left(R_{E}+h_{\mathrm{RX}}\right)^{2} \\
& -2\left(R_{E}+h_{\mathrm{TX}}\right)\left(R_{E}+h_{\mathrm{RX}}\right) \cos \left(\frac{d}{R_{E}}\right) .
\end{aligned}
$$

The angles $\alpha, \beta$ in Figure 1 are obtained by using

$$
\begin{aligned}
& \alpha+\beta=\frac{d}{R_{E}}=\frac{D \_1+D \_2}{R_{E}}, \\
& \arccos \left(\frac{R_{E}+h_{\mathrm{TX}}-R_{E} \cdot \cos \alpha}{D R \_1}\right)+\alpha \\
& =\arccos \left(\frac{R_{E}+h_{\mathrm{RX}}-R_{E} \cdot \cos \beta}{D R_{\_} X}\right)+\beta .
\end{aligned}
$$

Here, the lengths of $D R \_X 1$ and $D R \_X 2$ are as follows:

$$
\begin{aligned}
D R_{\_} X 1^{2}= & \left(R_{E}+h_{\mathrm{TX}}\right)^{2}+R_{E}^{2}-2\left(R_{E}+h_{\mathrm{TX}}\right) \cdot R_{E} \\
& \cdot \cos \alpha, \\
D R \_X 2^{2}= & \left(R_{E}+h_{\mathrm{RX}}\right)^{2}+R_{E}^{2}-2\left(R_{E}+h_{\mathrm{RX}}\right) \cdot R_{E} \\
& \cdot \cos \beta .
\end{aligned}
$$

Finally, the REL model [6] for open-sea radio propagation can be further expressed as follows:

$$
\begin{gathered}
\mathrm{PL}_{e}=20 \lg \left(\frac{\lambda}{4 \pi D}\right)+20 \lg (\eta)+L_{e_{-} \text {diff }}, \\
\eta=\left|1+R_{\text {rough }} \cdot S_{c} \cdot \operatorname{Div}_{\text {eff }} \cdot e^{j(2 \pi / \lambda) D_{\text {diff }}}\right|,
\end{gathered}
$$

where $R_{\text {rough }}$ represents effective reflection coefficient. $S_{c}$ and Div $_{\text {eff }}$ are corresponding to shadowing coefficient, and divergence coefficient, respectively. Considering the case of long TX-RX distance, $L_{e_{-} \text {diff }}$ denotes the diffraction loss derived from earth curvature effect. On the basis of (6), the structure of this propagation path loss model is divided into two parts (shown in Figure 2).

Free space model (Part-1) estimates the path loss derived from LOS ray between TX and RX. Also, this model can be 


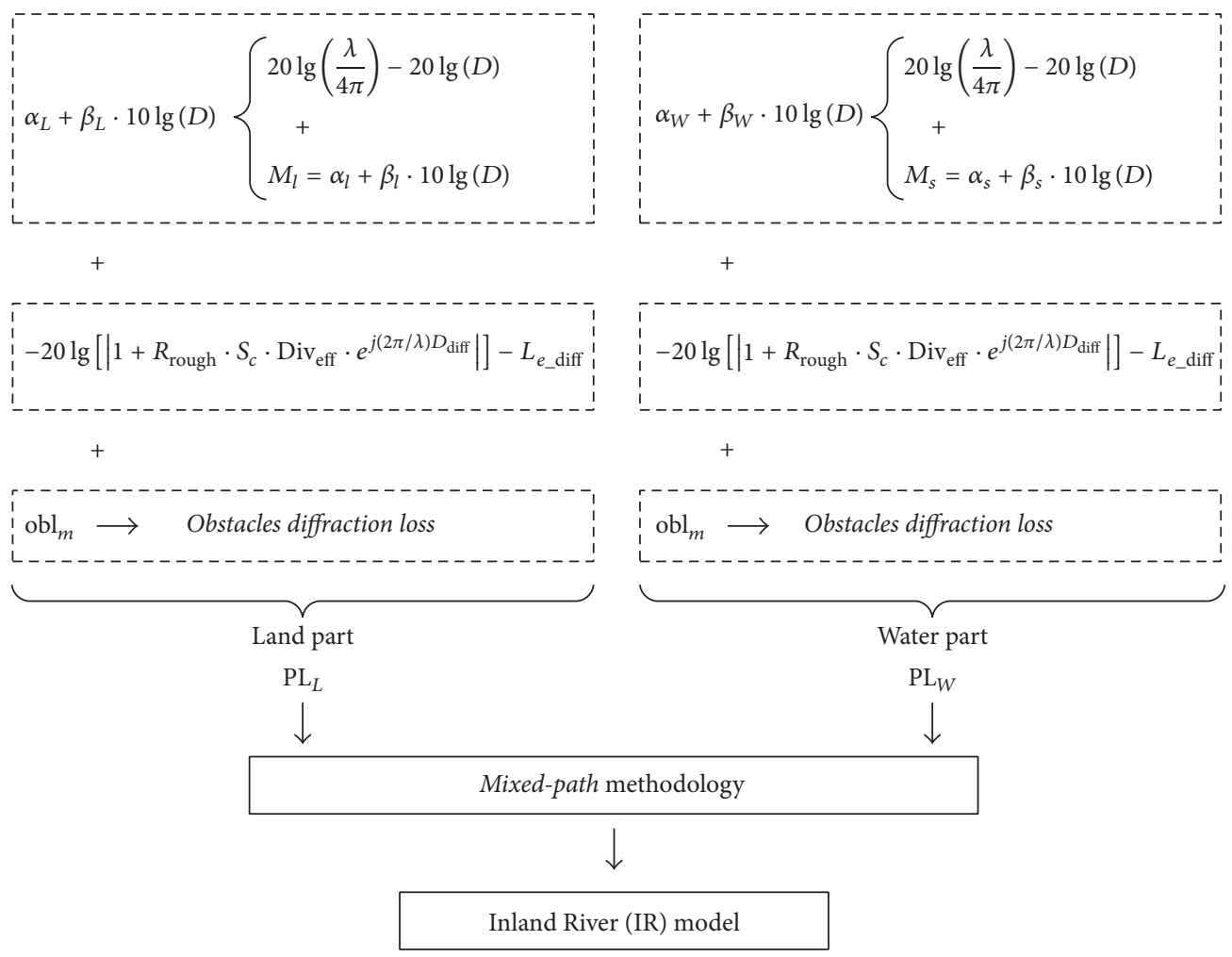

Figure 3: The structure of Inland River model.

transformed to a linear function of $\lg (D)$ [9]. For reflected signal, various effect factors such as effective reflection, shadowing, divergence, and earth curvature will increase the propagation path loss especially for the case at large TX-RX distances for the open sea [6]. Thus, Part-2 is an integration of them to make REL model applicable for the open sea environment.

2.2. Inland River Model. There are common features between sea surface and river surface. Therefore, scattering, shadowing, and divergence still need to be taken into consideration. With an increase of TX-RX distance, the diffraction loss caused by earth curvature will also occur. More details will be given in Section 4. However, inland river area which contains both land region and water region is different from the opensea, for example, buildings or bridges on the river bank may influence signal transmission. In addition, the conductivity of fresh water $\left(\sigma_{c}=0.001\right)$ is also different from sea water $\left(\sigma_{c}=4\right)$ [8]. In summary, compared to the REL model, there are three improvements in the IR model.

2.2.1. Parameters Optimization. Low matching degree of the REL model and free space model by comparing with the measurement data proves inefficiency of these classic models [10, 11], which indicate the necessity of model optimization. Since the inland river region is a combination of all-land region and all-water region, the corresponding radio propagation path loss for these single scenarios $\mathrm{PL}_{L}$ and $\mathrm{PL}_{W}$ are defined as the upper and lower bounds of the IR model. According to the Two-Ray theory, environmental correction factors $M_{l}$ and $M_{s}$ are employed to optimize IR model to obtain the $\mathrm{PL}_{L}$ and $\mathrm{PL}_{W}$, respectively. Assuming that both $M_{l}$ and $M_{s}$ in $\mathrm{dB}$ can also be depicted by linear format dependence with the logarithmic length of LOS path (shown in Figure 3), the parameters $\alpha_{L}, \beta_{L}$ for all-land region and the parameters $\alpha_{W}$, $\beta_{W}$ for all-water region are obtained by

$$
\begin{aligned}
& \alpha_{L}=20 \lg \left(\frac{\lambda}{4 \pi}\right)+\alpha_{l}, \\
& \beta_{L}=\beta_{l}-2 \\
& \alpha_{W}=20 \lg \left(\frac{\lambda}{4 \pi}\right)+\alpha_{s}, \\
& \beta_{W}=\beta_{s}-2,
\end{aligned}
$$

where $\alpha_{l}, \beta_{l}, \alpha_{s}$, and $\beta_{s}$ are empirical parameters. More details about these parameters' estimation will be given in Section 5 .

2.2.2. Diffraction Loss Caused by Obstacles near the River. Watercourse is narrow and sinuous. The buildings located at the river bank or some other facilities (e.g., bridges) may cause an additional diffraction loss $\mathrm{Obl}_{m}$ which is ignorable in open-sea environment. Thus, IR model includes this part shown in Figure 3. The calculation of this parameter can be found in Section 4.

2.2.3. Mixed-Path Methodology. Mixed-path feature for the inland river environment makes it necessary to combine the path losses of land region $\mathrm{PL}_{L}$ and water region $\mathrm{PL}_{W}$ (shown 


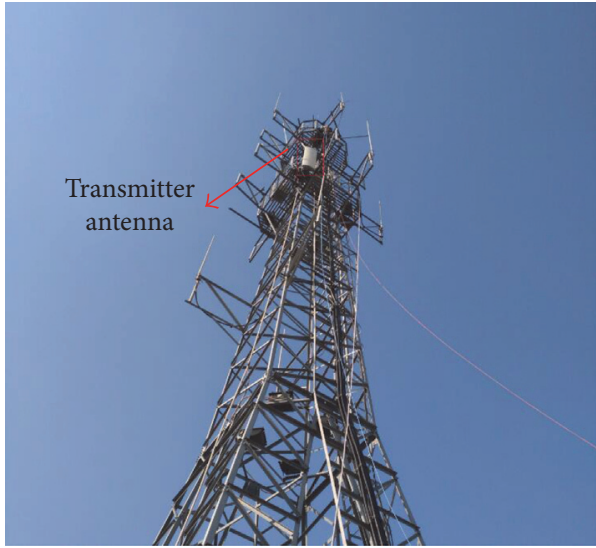

(a) Transmitter antenna on the iron tower in Jianghan district

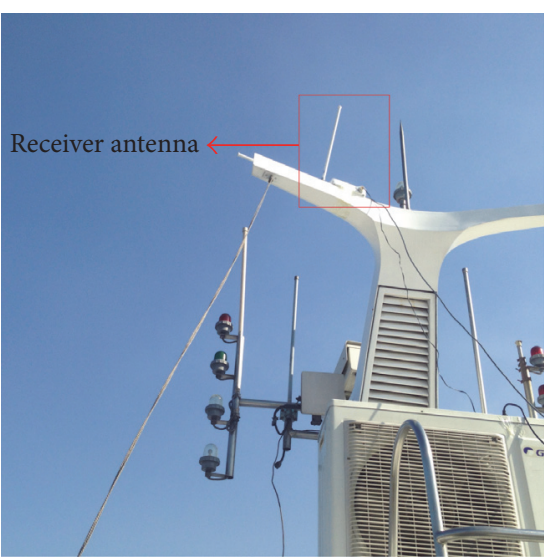

(b) Receiver antenna at the patrol boat

FiguRE 4: Transmitter antenna and receiver antenna.

in Figure 3). To resolve this problem, a proper method which is proposed by [7] is expressed as follows:

$$
\mathrm{PL}_{\mathrm{IR}}=(1-A) \cdot \mathrm{PL}_{L}+A \cdot \mathrm{PL}_{W} \text {. }
$$

Here, $\mathrm{PL}_{\mathrm{IR}}$ expresses the propagation path loss for inland river. " $A$ " is a mixed-path interpolation factor, which is given in (9). The percentage of the water path $F_{\text {river }}$ and the land path $F_{\text {land }}$ can be obtained from GPS position information in the route which is also recorded in our measurement data

$$
\begin{aligned}
& A=\left(1-\left(1-F_{\text {river }}\right)^{2 / 3}\right)^{V}, \\
& V=\max \left[1.0,1.0+\frac{\Delta}{40.0}\right], \\
& \Delta=E_{\text {land }}(D)-E_{\text {river }}(D) .
\end{aligned}
$$

In (11), $E_{\text {land }}$ and $E_{\text {river }}$ correspond to the equivalent field strength $(\mathrm{dB})$ for all-land and all-water paths, respectively:

$$
\begin{aligned}
& E_{\text {land }}(D)=139.3-\mathrm{PL}_{L}+20 \lg (f) \\
& E_{\text {river }}(D)=139.3-\mathrm{PL}_{W}+20 \lg (f)
\end{aligned}
$$

Here, $f$ denotes the carrier frequency in $\mathrm{MHz}$.

\section{Measurement Campaigns}

In our project, measurement campaigns were performed for three different scenarios described as follows:

(i) Urban watercourse environment with few obstacles between TX and RX

(ii) Urban watercourse environment with huge buildings between TX and RX

(iii) Suburban watercourse environment

In all of these measurement campaigns, Zhongxing Telecommunication Equipment Corporation (ZTE) provided most of apparatuses, which contain cross-polarized base station (BS) antenna (TX), Customer Premise Equipment (CPE), Building Baseband Unit (BBU), and Remote Radio Unit (RRU), which operate in the frequency range from $1447 \mathrm{MHz}$ to $1467 \mathrm{MHz}$. The transmitter end emitted 4G signal with $20 \mathrm{MHz}$ bandwidth. Considering the case of long distance transmission, uplink-downlink and special subframe were set to configuration 1 and configuration 7 [12] during the whole tests, respectively. In addition, both GPS position data and the information of vessel speed were collected and saved through a Motorola mobile phone (ME525).

3.1. Urban Environment Measurement Campaign Description. Figure 4(a) shows that RRU and transmitter antenna $(15 \mathrm{dBi}$ gain, $65^{\circ}$ (Az.) and $8^{\circ}$ (El.)) were placed on the iron tower which was located at the roof of a tall building near the river. Therefore, the height of BS antenna is equal to $79.55 \mathrm{~m}$ (including the height of iron tower: $68.30 \mathrm{~m}$, the length of TX antenna: $1.50 \mathrm{~m}$, and the height of shore: $9.75 \mathrm{~m}$ ) above the river level after ignoring the influence of season tide. At the receiver side (Figure 4(b)), the CPE connected to the PC via RJ45 port can both transmit and receive the radio signals through two omnidirectional antennas ( $3.5 \mathrm{dBi}$ gain). After that, our urban measurement campaigns can be divided into downstream part and upstream part as follows.

3.1.1. Downstream Part. The patrol boat sailed from a wharf beside our base station to the Tianxingzhou Yangtze River Bridge with the average speed of 14 knots. There was no obstacle higher than TX in this direction. Moreover, two bridges (shown in Figure 5) which can be found between the BS and terminal point were the Second Wuhan Yangtze River Bridge $(3.511 \mathrm{~km}$ away from the TX) and Erqi Yangtze River Bridge (6.8 $\mathrm{km}$ away from the TX), respectively.

3.1.2. Upstream Part. Using the same BS and ship, we completed a $15.01 \mathrm{~km}$ measurement campaign in the upstream 


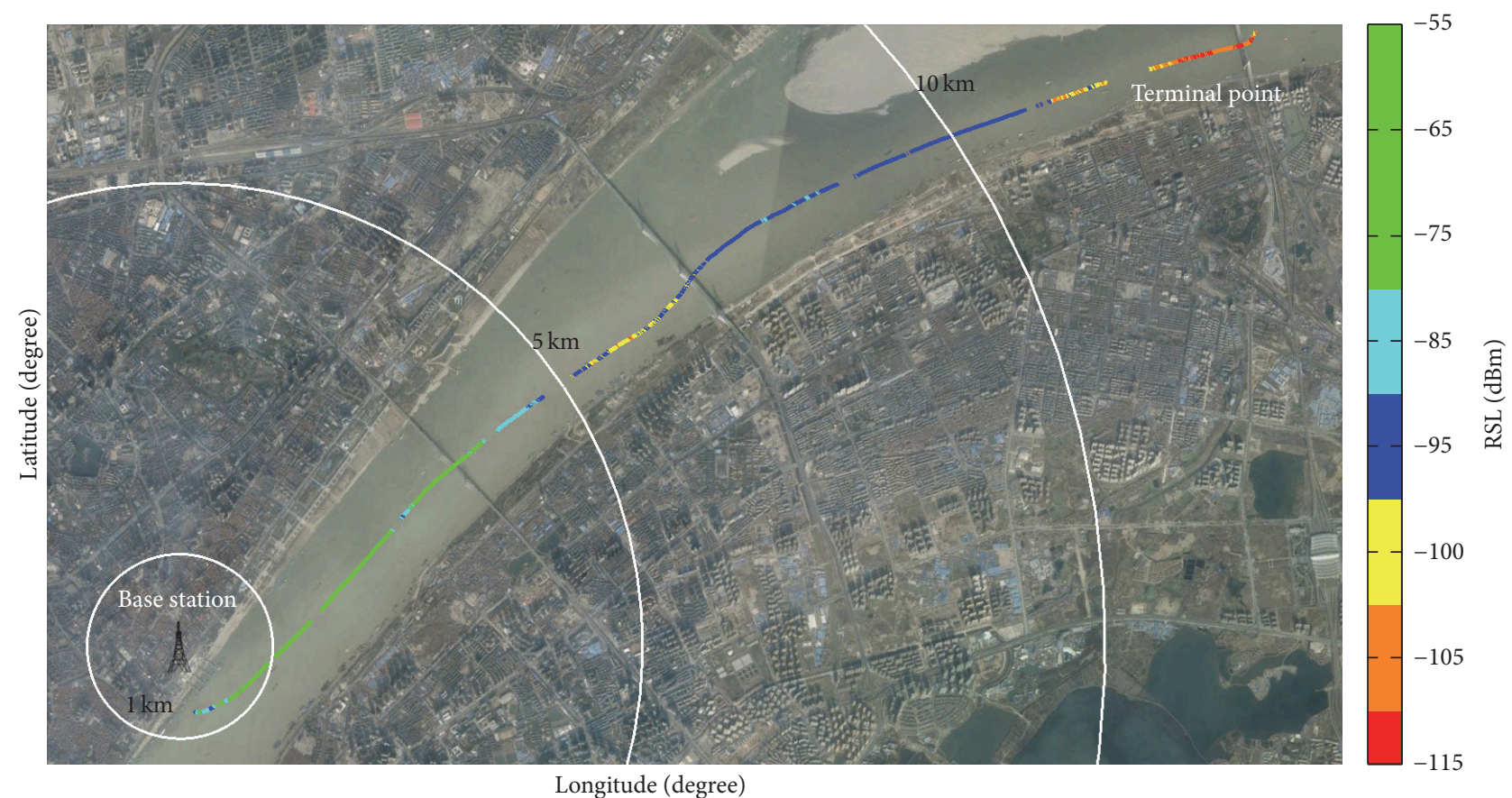

FIGURE 5: The route of measurement campaign 1 with few obstacles between TX and RX.

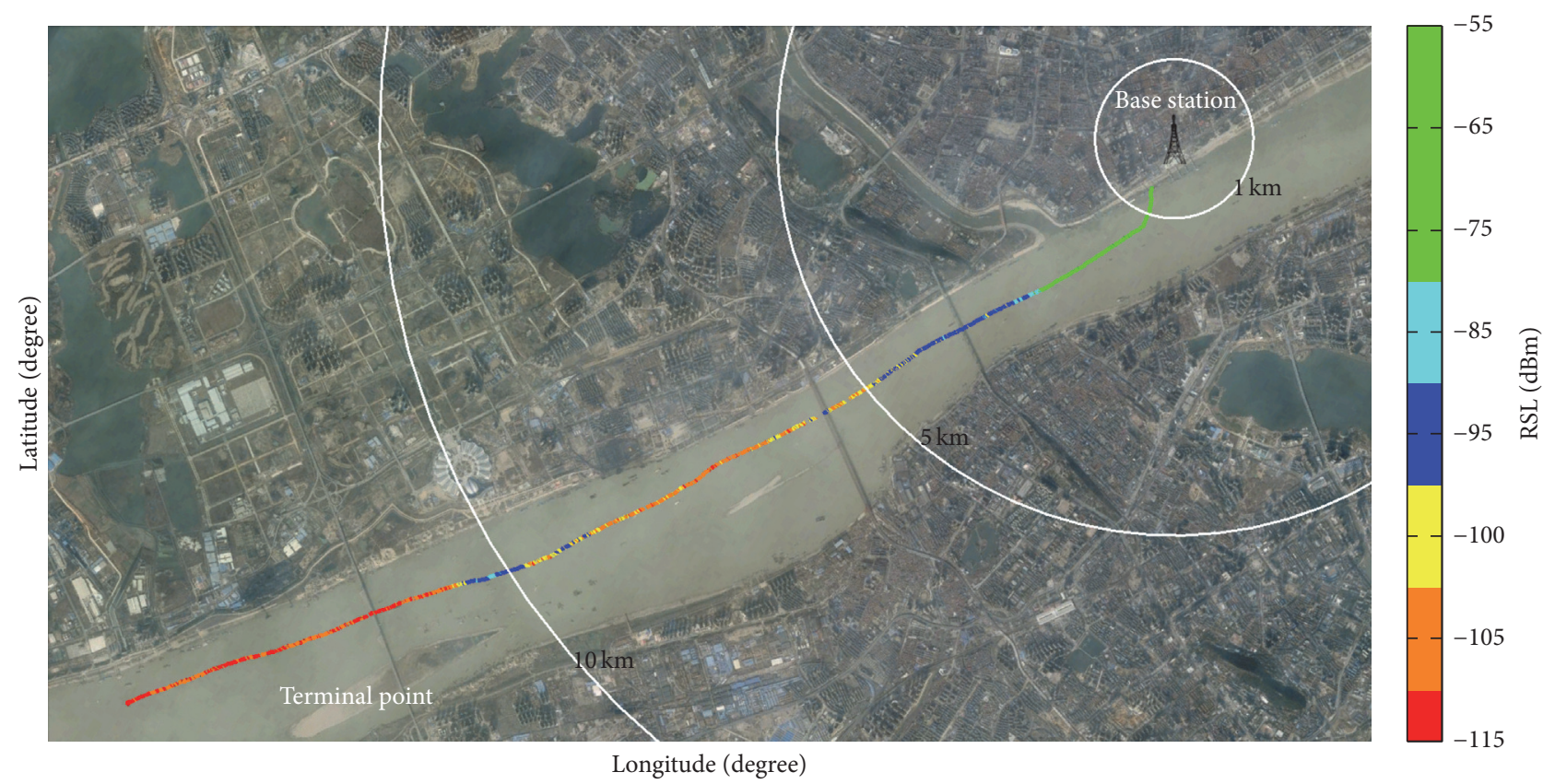

FIGURE 6: The route of measurement campaign 2 with large buildings between TX and RX.

direction (shown in Figure 6). The average speed was approximately 13 knots during the whole process. It was different from the downstream environment since there were some huge buildings (average height: $157 \mathrm{~m}$ ) at the distance of $700 \mathrm{~m}$ from our base station.
3.2. Suburban Environment Measurement Campaign Description. As shown in Figure 7, this measurement campaign was conducted in a suburban area which was positioned at Dengjiakou Town. The effective height of our BS antenna (TX) was $53.00 \mathrm{~m}$ (including the height of iron tower: $50.00 \mathrm{~m}$, the 


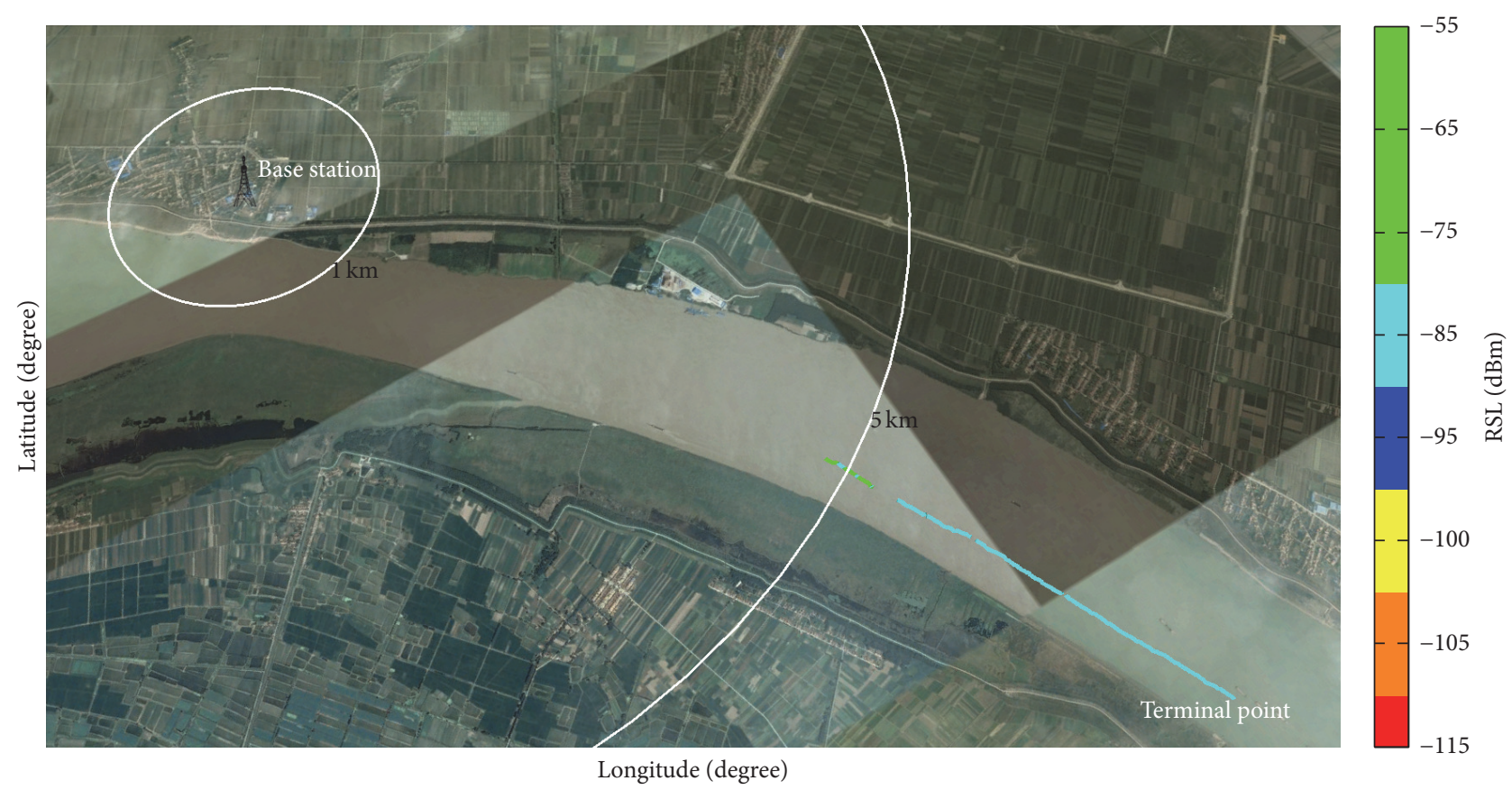

FIgURE 7: The route of suburban measurement campaign.

length of TX antenna: $1.50 \mathrm{~m}$ and the height of shore: $1.50 \mathrm{~m}$ ) above river level. Due to the supervision region limitation, our patrol boat with the CPE sailed only $3 \mathrm{~km}$ for this scenario.

Ultimately, all of the measurement information is summarized in Table 1.

\section{Effect Factors Analysis}

As mentioned above, the IR model contains some effect factors which will be analyzed in the following contents.

4.1. Finite Dimensions Diffraction Loss. The diffraction loss caused by obstacles (such as huge buildings and bridges) along the river is an important effect factor. Many approximate methods based on multiple knife-edges diffraction have been proposed to calculate its loss value (e.g., Bullington method [2], Epstein-Petersen method [13], Deygout method [14], ITU-R method [15], etc.). To make such calculations, an assumption that the two sides of an obstacle are regarded as two thin screens of negligible thickness is necessary [15]. In addition, Professor Molisch [1] provided a comparison of these different methods. According to measurement campaigns, Deygout method is an appropriate one due to inland river environment fulfills its requirement: "small number of screens of different height."

In Figure 8(a), each building is simplified as two infinitewidth screens which corresponds its two sides. However, the idealized infinite-width screen will increase the diffraction loss in most instances because the width of obstacles actually is finite. Thus, the finite-width screen diffraction given by paper [15-17] for analyzing finite dimensions obstacles will be employed in this paper. The approach can be regarded as a combination of multiple knife-edges diffraction in several directions (e.g., three-knife-edges diffraction consists of top, left side, and right side, as shown in Figure 8(b)) [15].

According to the description of Section 3.1, there is a bridge which is $3.511 \mathrm{~km}$ away from the base station. The corresponding special finite-width obstacle diffraction can be simulated in Figure 9. Two parts diffraction loss should be calculated in this case.

4.1.1. The Diffraction above the Bridge. Figure 10 provides the geometrical information based on measurement 1. After that, diffraction loss $L_{\text {on }}$ is given by

$$
\begin{aligned}
L_{\text {on }} & =-20 \lg \left(\left|\frac{1}{2}-\frac{e^{(j \cdot \pi / 4)}}{\sqrt{2}} \cdot F\left(v_{f_{\text {_up }}}\right)\right|\right)+L_{02}, \\
F\left(v_{f_{\text {_up }}}\right) & =\int_{0}^{v_{f \text { up }}} e^{\left(-j \cdot \pi\left(t^{2} / 2\right)\right)} d t,
\end{aligned}
$$

where $v_{f_{\text {_up }}}$ is the Fresnel parameter. According to [1], the screen 02 (shown in Figure 10) is regarded as "main screen" due to a higher single knife-edge diffraction loss $L_{02}$ :

$$
\begin{aligned}
v_{f_{\text {_up }}}= & \theta_{\mathrm{on}} \sqrt{\frac{2}{\lambda \cdot\left(d_{\text {t2ob1 }}^{-1}+W_{b}^{-1}\right)}} \\
\theta_{\mathrm{on}}= & \arctan \left(\frac{\left(\left(h_{b}+h_{b \mathrm{th}}\right)-h_{\mathrm{TX}}\right)}{d_{t 2 \mathrm{ob} 1}}\right) \\
& +\arctan \left(\frac{\left(\left(h_{b}+h_{b \mathrm{th}}\right)-\left(h_{b}+h_{b \mathrm{th}}\right)\right)}{W_{b}}\right) .
\end{aligned}
$$

In (14), $\theta_{\text {on }}$ denotes the diffraction angle of screen $01 . h_{b \text { th }}$ is the thickness of obstacle. $h_{b}, W_{b}, d_{t 2 \mathrm{ob} 1}$, and $h_{\mathrm{TX}}$ represent 
TABLE 1: The measurement parameters.

\begin{tabular}{lccc}
\hline Parameters & Data_1 & Data_2 & Data_3 \\
\hline Carrier frequency & $1457 \mathrm{MHz}$ & $1457 \mathrm{MHz}$ & $1457 \mathrm{MHz}$ \\
Bandwidth & $20 \mathrm{MHz}$ & $20 \mathrm{MHz}$ & $20 \mathrm{MHz}$ \\
TX power & $49.8 \mathrm{dBm}$ & $49.8 \mathrm{dBm}$ & $49.8 \mathrm{dBm}$ \\
TX beam width & $\left(65^{\circ} \mathrm{Az} .8^{\circ} \mathrm{El}\right)^{*}$ & $\left(65^{\circ} \mathrm{Az} .8^{\circ} \mathrm{El} .\right)^{*}$ & $\left(65^{\circ} \mathrm{Az} .8^{\circ} \mathrm{El}^{*}\right)^{*}$ \\
RX beam width & Omni & Omni & Omni \\
TX height & $79.55 \mathrm{~m}$ & $79.55 \mathrm{~m}$ & $53.00 \mathrm{~m}$ \\
RX height & $3.4 \mathrm{~m}$ & $3.4 \mathrm{~m}$ & $3.4 \mathrm{~m}$ \\
TX gain & $15 \mathrm{dBi}$ & $15 \mathrm{dBi}$ & $15 \mathrm{dBi}$ \\
RX gain & $3.5 \mathrm{dBi}$ & $3.5 \mathrm{dBi}$ & $3.5 \mathrm{dBi}$ \\
Max.distance & $11.06 \mathrm{~km}$ & $15.01 \mathrm{~km}$ & $7.778 \mathrm{~km}$ \\
Temperature & $8-18^{\circ} \mathrm{C}$ & $8-18^{\circ} \mathrm{C}$ & $11-21^{\circ} \mathrm{C}$ \\
File size & $6.204 \mathrm{~GB}$ & $6.204 \mathrm{~GB}$ & $6.204 \mathrm{~GB}$ \\
\hline
\end{tabular}

${ }^{*}$ Sector antenna.

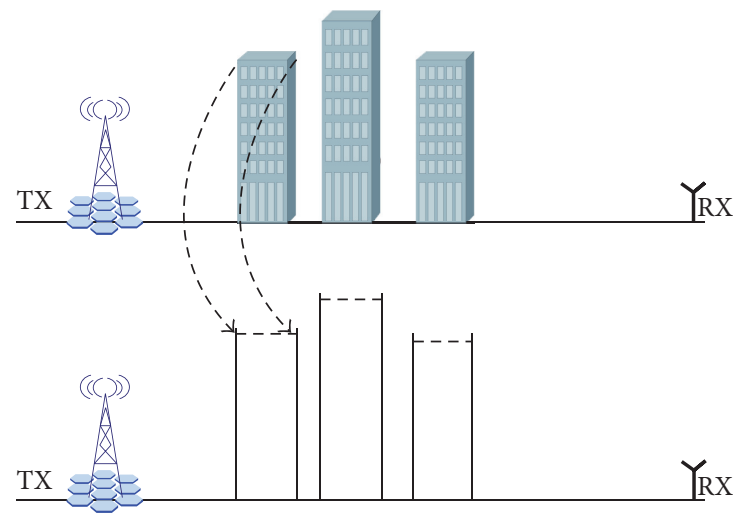

(a) Obstacles approximation

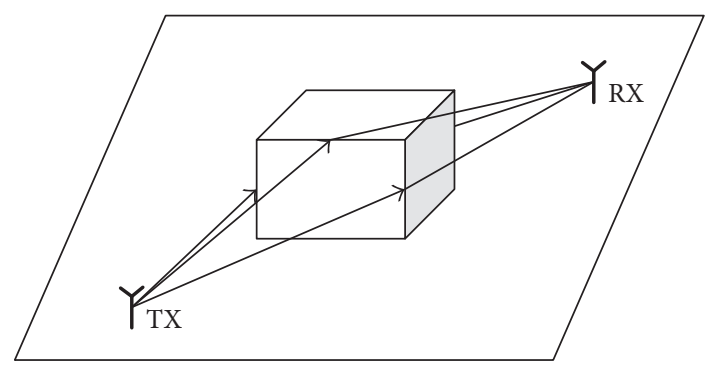

(b) Finite dimensions obstacle diffraction

FIGURE 8: Obstacles approximation and finite dimensions obstacle diffraction.

bridge's height, bridge's width, and distance from TX to screen 01 and the height of TX, respectively.

4.1.2. The Diffraction Loss under the Bridge. The diffraction loss under the bridge $L_{\text {down }}$ is calculated by using

$$
\begin{aligned}
L_{\text {down }}= & -20 \lg \left(\left|\frac{1}{2}-\frac{e^{(j \cdot \pi / 4)}}{\sqrt{2}} \cdot F\left(v_{f_{- \text {down }}}\right)\right|\right) \\
& +l_{01}, \\
F\left(v_{f_{\text {ddown }}}\right)= & \int_{0}^{v_{f \text { down }}} e^{\left(-j \cdot \pi\left(t^{2} / 2\right)\right)} d t,
\end{aligned}
$$

where $v_{f \text { down }}$ is the Fresnel parameter in this case, which can be obtained by using (16). Unlike the diffraction part above the bridge, the screen 01 (shown in Figure 11) results in a larger diffraction loss $l_{01}$ than the screen 02 :

$$
v_{f_{\text {_down }}}=\theta_{\text {under }} \sqrt{\frac{2}{\lambda \cdot\left(d_{\mathrm{ob} 2 \_2 r}^{-1}+W_{b}^{-1}\right)}} .
$$

Here, $d_{\mathrm{ob} 22 r}$ denotes the distance between screen 02 to RX. The $\theta_{\text {under }}$ is given by

$$
\begin{aligned}
\theta_{\text {under }}= & \arctan \left(\frac{\left(\left(h_{\mathrm{TX}}-h_{b}\right)-\left(h_{\mathrm{TX}}-h_{b}\right)\right)}{W_{b}}\right) \\
& +\arctan \left(\frac{\left(\left(h_{\mathrm{TX}}-h_{b}\right)-\left(h_{\mathrm{TX}}-h_{\mathrm{RX}}\right)\right)}{d_{\mathrm{ob} 22 r}}\right) .
\end{aligned}
$$

The $h_{\mathrm{RX}}$ which can be found in Figure 11 denotes the RX antenna height. According to [15], the total bridge diffraction loss is expressed as

$$
\begin{aligned}
\xi_{\text {on }}(\vartheta) & =10^{\left(L_{\text {on }} / 20\right)}, \\
\xi_{\text {down }}(\vartheta) & =10^{\left(L_{\text {down }} / 20\right)}, \\
\operatorname{Dif}_{\text {mean }}(\vartheta) & =-10 \cdot \lg \left(\xi_{\text {on }}^{-2}(\vartheta)+\xi_{\text {down }}^{-2}(\vartheta)\right),
\end{aligned}
$$

where $\xi_{\text {on }}$ and $\xi_{\text {down }}$ are the loss factors. Dif ${ }_{\text {mean }}$ denotes the average diffraction loss of the bridge. Since the Second Yangtze River Bridge is a suspension bridge, the constructions on it cannot be ignored. Therefore, screen 01 and screen 02 


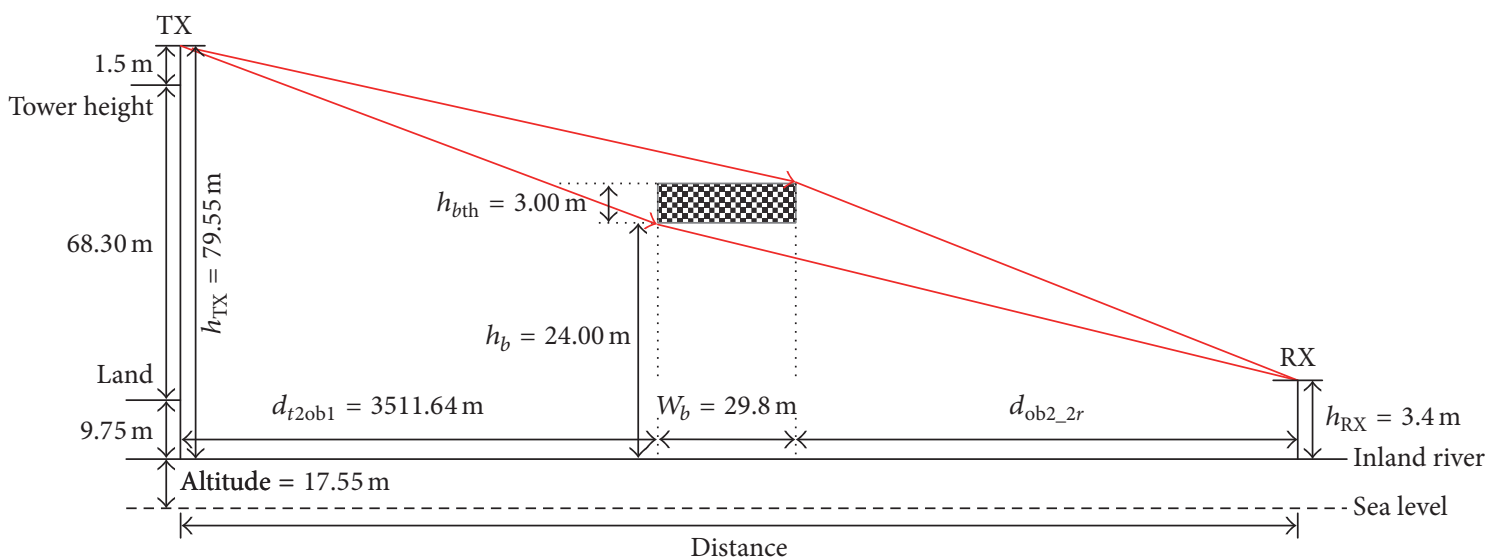

Figure 9: Finite dimensions bridge diffraction.

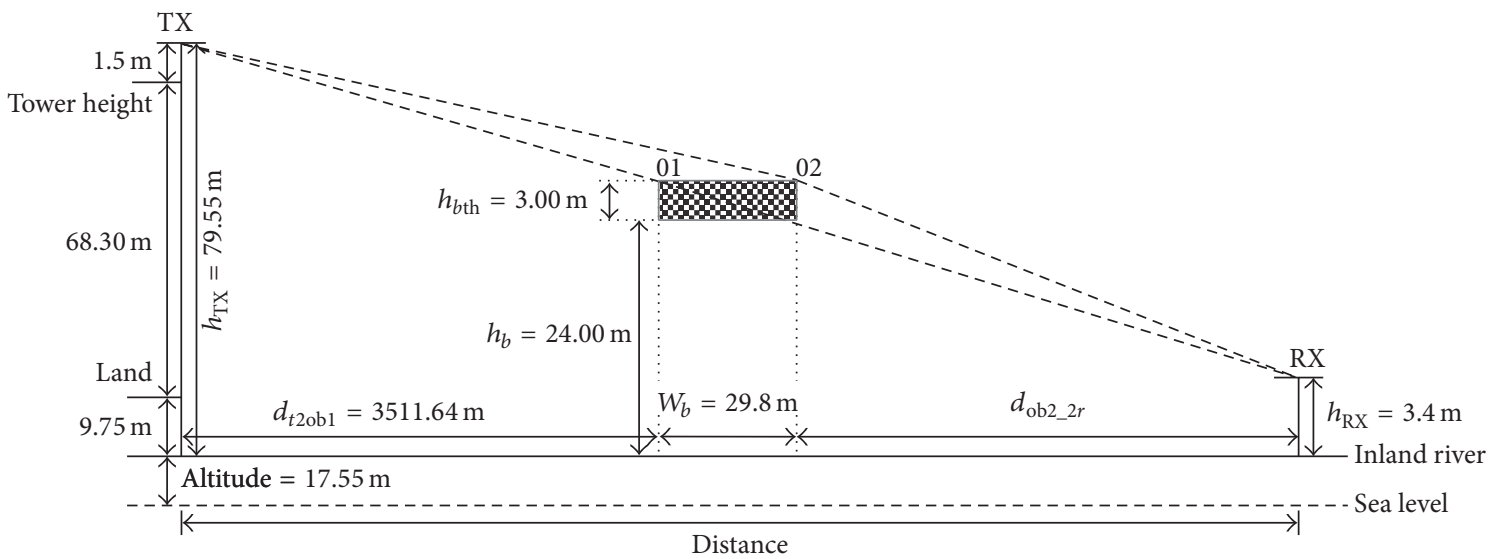

Figure 10: Diffraction over the bridge.

shown in Figures 10 and 11 should also include the height of obstacles. On the basis of measurement data, the diffraction loss caused by this bridge is shown in Figure 12.

The results indicate that there is a large increase of diffraction up to $18 \mathrm{~dB}$ loss between $4 \mathrm{~km}$ and $6 \mathrm{~km}$. Its maximum value occurs at a TX-RX distance of $6.5 \mathrm{~km}$ away from the BS. The corresponding diffraction loss maintains within $[0,5 \mathrm{~dB}]$ when the TX-RX distance is longer than $9 \mathrm{~km}$.

In measurement campaign 2, there are some high buildings with an average height of $157 \mathrm{~m}$ at a TX-RX distance of $700 \mathrm{~m}$. The finite dimensions obstacles' diffraction for this scenario can be approximated as an equivalent structure in Figure 13. Similar estimation approach is used to acquire their diffraction loss.

As shown in Figures 14 and 15, obstacle 1 will influence the measurement route ranging from $3 \mathrm{~km}$ to $10 \mathrm{~km}$ while obstacle 2 will bring additional diffraction loss between $10 \mathrm{~km}$ and $15 \mathrm{~km}$. Thus, a diffraction loss dip should be found at the distance of $10 \mathrm{~km}$, which is caused by a $40.1 \mathrm{~m}$ space between obstacle 1 and obstacle 2 .

For measurement campaign 3 in suburban environment, the diffraction loss can be neglected $\left(\mathrm{Obl}_{m}=0 \mathrm{~dB}\right)$ because the height of base station is much higher than the other buildings nearby.

4.2. Other Effect Factors. IR model inherits some effect factors from the REL model due to their similarity of environment. All of them will be described in detail as follows.

4.2.1. Effective Reflection from River Surface. The inland river surface is rarely smooth due to the water roughness (shown in Figure 16). As a result, the scattering leads to an energy reduction compared with the idealize specular reflection [6]. Assuming the height distribution of the water surface in inland river is similar with the sea wave surface which agrees with a Gaussian distribution [18]. The effective reflection coefficient $R_{\text {rough }}$ is given by

$$
\begin{aligned}
& R_{\text {rough }}=R \cdot e^{-2 \cdot\left((2 \pi / \lambda) \sigma_{h} \sin \left(\pi / 2-\theta_{\mathrm{IN}}\right)\right)^{2}}, \\
& \mathrm{Eff}_{\mathrm{TX}}=h_{\mathrm{TX}}-0.5 \cdot R_{E} \cdot \alpha^{2}, \\
& \mathrm{Eff}_{\mathrm{RX}}=h_{\mathrm{RX}}-0.5 \cdot R_{E} \cdot \beta^{2},
\end{aligned}
$$

where $\theta_{\mathrm{IN}}$ denotes the incident angle in Figure 1. Eff $\mathrm{TX}$ and $\mathrm{Eff}_{\mathrm{RX}}$ correspond to the effective height of TX and RX 


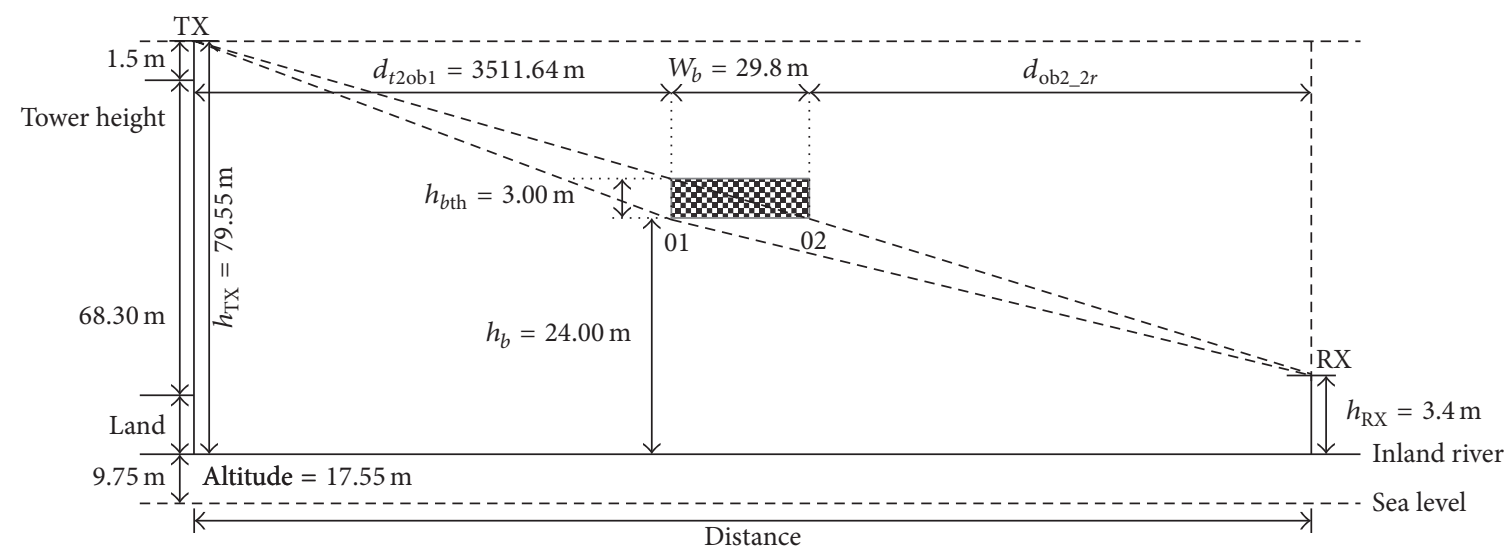

Figure 11: Diffraction under the bridge.

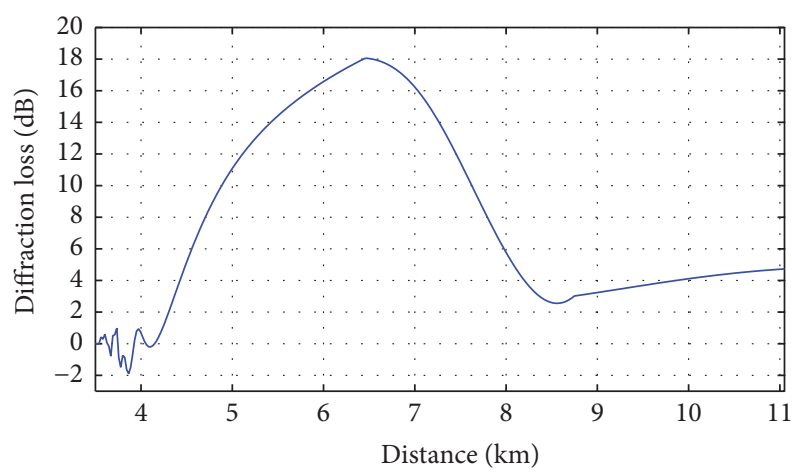

Figure 12: Diffraction loss caused by bridge in measurement 1 .

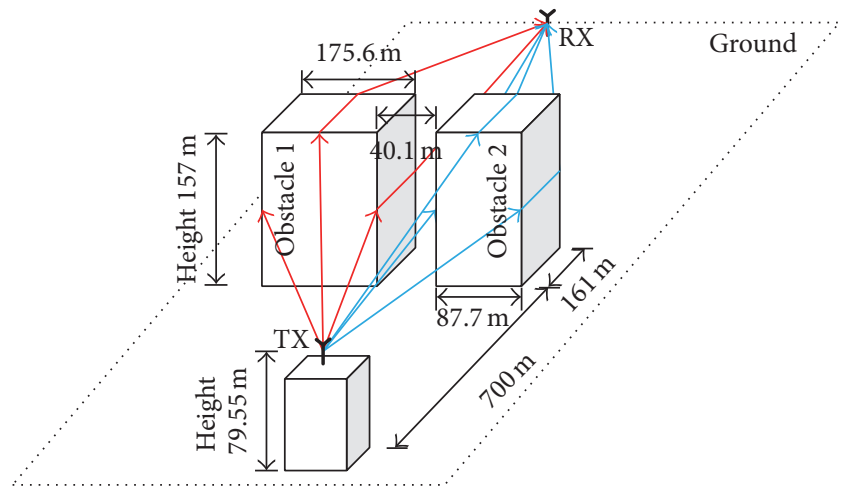

FIGURE 13: Finite dimensions buildings diffraction.

antennas, respectively. $\sigma_{h}$ represents the standard deviation of surface height distribution. With increasing distance of TX and RX, the grazing angle $\phi\left(\phi=\pi / 2-\theta_{\mathrm{IN}}\right)$ reduces accordingly and approaches to zero when the radio link is tangent to the horizon. In this situation, the effective reflection coefficient $R_{\text {rough }}$ will approximate to the specular reflection coefficient $R$. According to [8], the value of $R$ is determined by permittivity $\varepsilon_{s}$ and conductivity $\sigma_{c}$ for various terrain types. Thus, the specular reflection coefficient of the

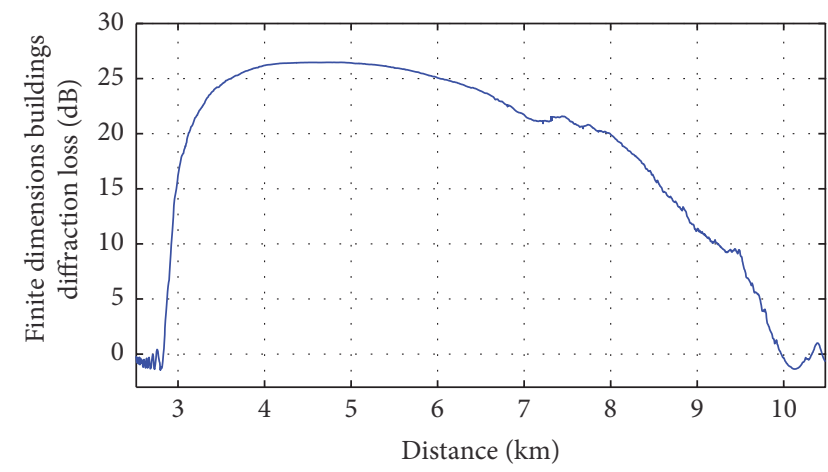

Figure 14: Diffraction loss caused by obstacle 1 in measurement 2.

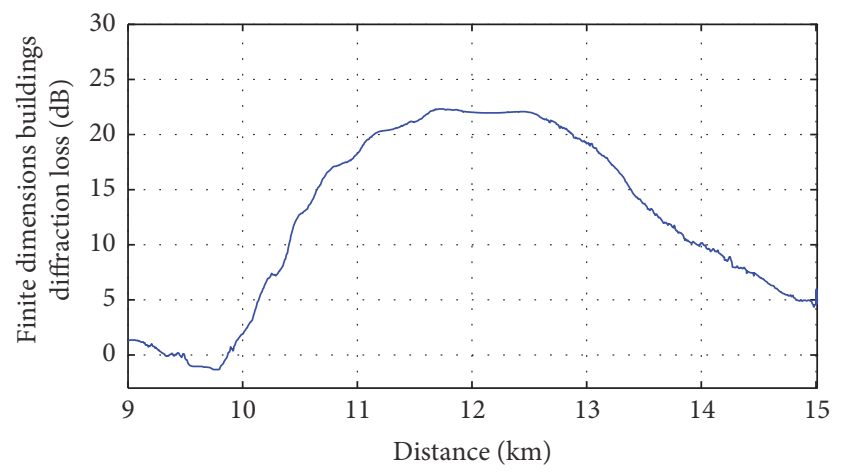

Figure 15: Diffraction loss caused by obstacle 2 in measurement 2.

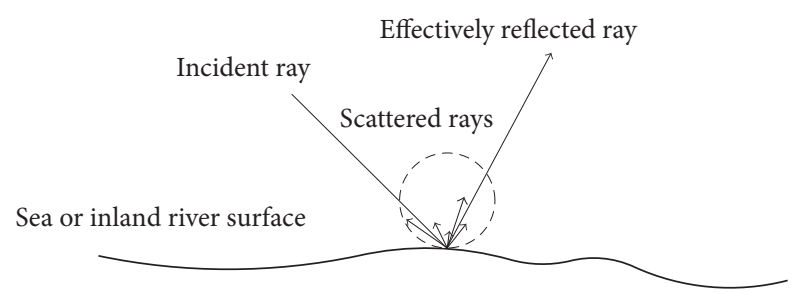

FIGURE 16: Scattering and effective reflection. 


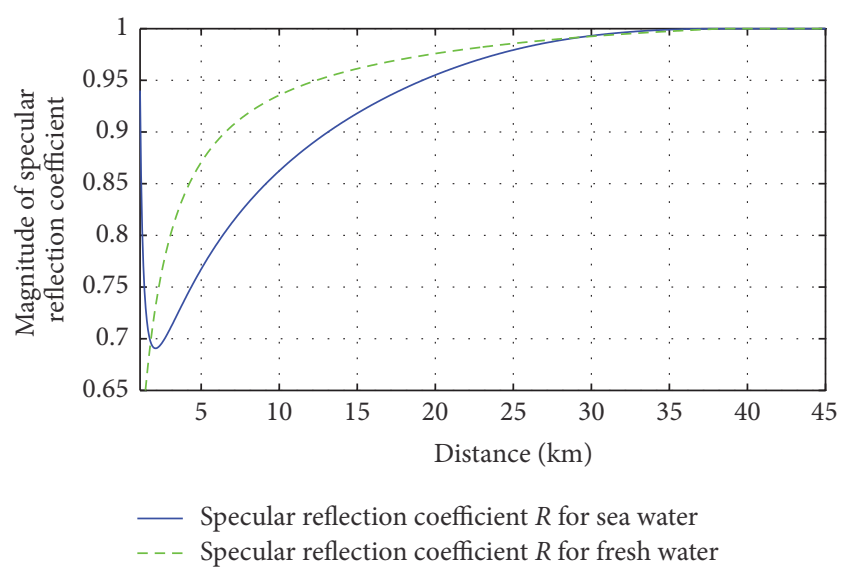

FIgURE 17: $R$ as a function of the TX-RX distance $\left(h_{\mathrm{TX}}=79.55 \mathrm{~m}\right.$, $\left.h_{\mathrm{RX}}=3.4 \mathrm{~m}\right)$.

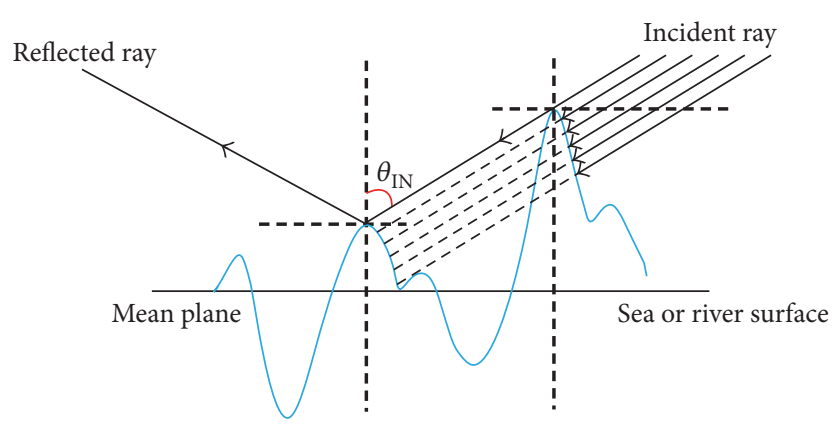

FIGURE 18: Shadowing effect on the reflected ray.

sea water $\left(\varepsilon_{s}=80, \sigma_{c}=4\right)$ is different from fresh water $\left(\varepsilon_{s}=\right.$ $80, \sigma_{c}=0.001$ ) of inland river (shown in Figure 17).

4.2.2. Shadowing Effect on the Reflected Ray. Although Kirchhoff Theory assumes that any point on the surface does not block others [1], the shadowing may still occur during the measurement campaigns, especially when the incident angle $\theta_{\mathrm{IN}}$ is large enough (shown in Figure 18). According to [19], this phenomenon should be considered when the elevation angle $\theta_{t c a}$ (shown in Figure 1) is less than 0.5 degrees. Afterwards, the shadowing coefficient $S_{c}$ based on the assumption that the sea surface and its slope can be modeled by using a two-dimensional Gaussian process is calculated by

$$
\begin{aligned}
S_{c} & =\frac{1-0.5 \cdot \operatorname{erfc}\left(\cot \theta_{\mathrm{IN}} / \sqrt{2} w_{0}\right)}{\Lambda+1} \\
\Lambda & =\frac{1}{2}\left(\sqrt{\frac{2}{\pi}} \cdot \frac{w_{0}}{\cot \theta_{\mathrm{IN}}} \cdot e^{\left(-\cot ^{2} \theta_{\mathrm{IN}} /\left(2 \cdot w_{0}^{2}\right)\right)}\right. \\
& \left.-\operatorname{erfc}\left(\frac{\cot \theta_{\mathrm{IN}}}{\sqrt{2} w_{0}}\right)\right)
\end{aligned}
$$

where erfc is an error function and $w_{0}^{2}$ denotes the meansquare surface slope [20]. The shadowing coefficient with

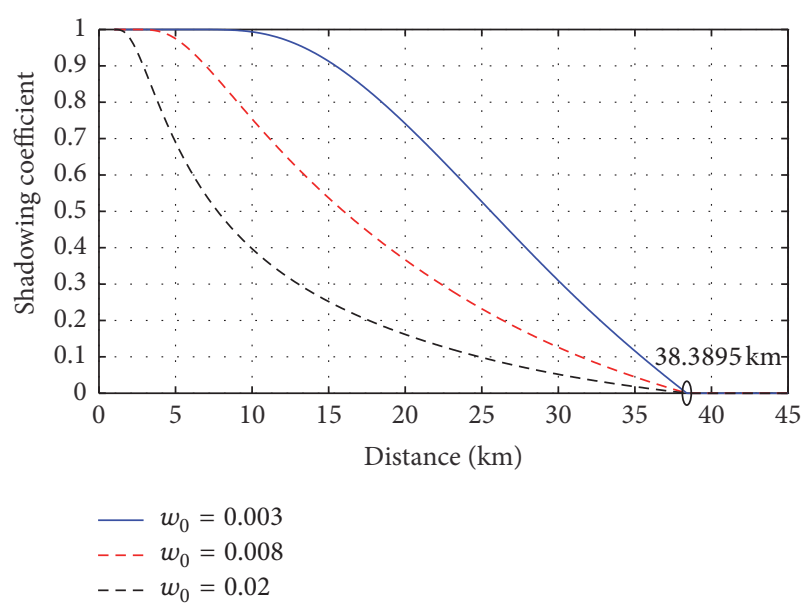

FIgURE 19: $S_{c}$ as a function of the TX-RX distance $\left(h_{\mathrm{TX}}=79.55 \mathrm{~m}\right.$, $\left.h_{\mathrm{RX}}=3.4 \mathrm{~m}\right)$.

different $w_{0}^{2}$ can be found in Figure 19. The figure illustrates that a larger value of $w_{0}$ will lead to a smaller shadowing coefficient. Moreover, all of $S_{c}$ are equal to zero because reflected rays are totally shadowed when the TX-RX distance exceeds $38.3895 \mathrm{~km}$.

4.2.3. Divergence. The earth curvature can decrease the power density $\rho_{E}$ carried by the reflected ray as shown in Figure 20. The reduction of $\rho_{E}$ will lead to a lower received signal level (RSL) [6]. Consequently, the divergence

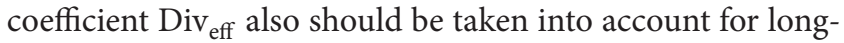
distance inland river path loss model:

$$
\begin{aligned}
& \operatorname{Div}_{\text {eff }} \\
& = \begin{cases}\frac{1}{\sqrt{1+\left(2 \cdot D \_1 D \_2\right) / R_{E}\left(E H_{1}+E H_{2}\right)}} ; & E H_{1}>0, E H_{2}>0 \\
0 ; & \text { otherwise, }\end{cases}
\end{aligned}
$$

where D_1 and D_2 are shown in Figure $1 . E H_{1}$ and $E H_{2}$ represent $\mathrm{Eff}_{\mathrm{TX}}$ and $\mathrm{Eff}_{\mathrm{RX}}$, respectively. Figure 21 indicates that the influence of $\mathrm{Div}_{\text {eff }}$ will become bigger with the increasing distance $d$. Ultimately, divergence coefficient also turns to zero beyond the distance $38.3895 \mathrm{~km}$.

4.2.4. Earth Curvature Effect. For the long-distance scenario, the surface of earth can be regarded as an obstacle which will block 0.6 First Fresnel Zone (FFZ) $D_{06}\left(f, h_{\mathrm{TX}}, h_{\mathrm{RX}}\right)$ between TX and RX antennas:

$$
\begin{aligned}
& D_{06}=\frac{D_{f} \cdot D_{h}}{D_{f}+D_{h}} \mathrm{~km}, \\
& D_{f}=3.89 \cdot 10^{-5} \cdot f h_{\mathrm{TX}} h_{\mathrm{RX}}, \\
& D_{h}=4.1\left(\sqrt{h_{\mathrm{TX}}}+\sqrt{h_{\mathrm{RX}}}\right) .
\end{aligned}
$$

References [21, 22] propose a theory of ground-wave propagation over a smooth spherical earth (shown in Figure 22), which is able to analyze the diffraction loss caused by 


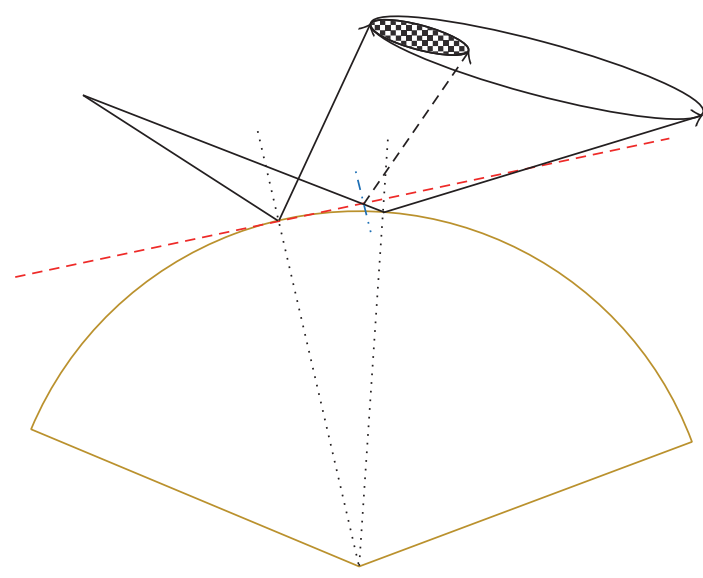

FIGURE 20: Divergence effect.

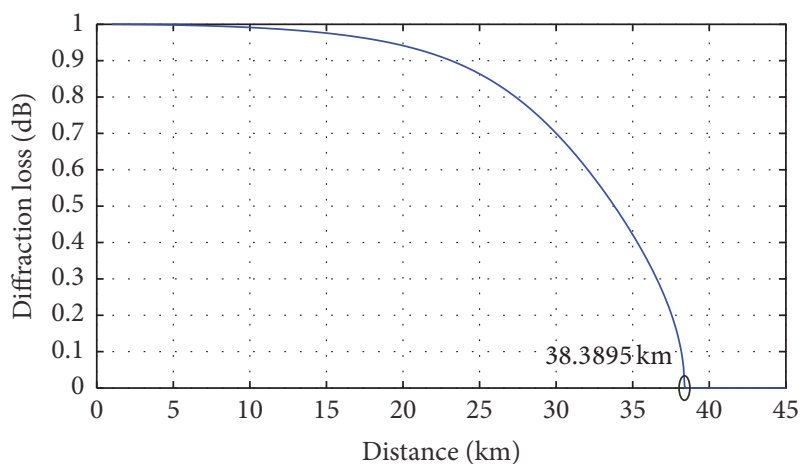

FIGURE 21: Divergence coefficient as a function of the TX-RX distance $\left(h_{\mathrm{TX}}=79.55 \mathrm{~m}, h_{\mathrm{RX}}=3.4 \mathrm{~m}\right)$.

earth curvature effect. The $d_{\mathrm{TXh}}$ denotes the distance between TX and the horizon while $d_{\mathrm{RXh}}$ represents the distance between RX and the horizon. Both of them can be obtained by

$$
\begin{aligned}
& \text { Dis_1 }=d_{\mathrm{TXh}}=\sqrt{2 k_{e} R_{E} \cdot h_{\mathrm{TX}}}, \\
& \text { Dis_2 }=d_{\mathrm{RXh}}=\sqrt{2 k_{e} R_{E} \cdot h_{\mathrm{RX}}}, \\
& d_{\text {sum }}=\text { Dis_1 }+ \text { Dis } 2,
\end{aligned}
$$

where $k_{e}$ is equal to 1 [19]. The TX-RX distance $d$ can be divided into Dis_1, Dis_2, and Dis_3 shown in Figure 22. Then the corresponding diffraction loss of each part is given as follows [6]:

$$
\begin{aligned}
& L_{1}=20 \lg \left(\frac{N_{1}}{\sqrt{5.656 \pi \zeta_{1}}}\right), \\
& L_{2}=20 \lg \left(N_{2}\right),
\end{aligned}
$$

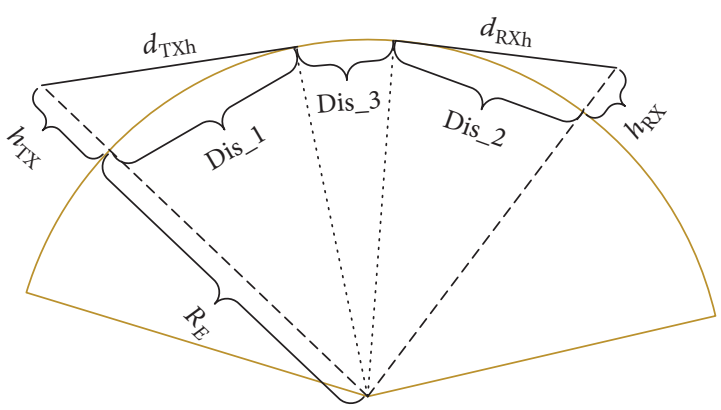

FIgURE 22: Ground-wave propagation over a smooth spherical earth.

$$
\begin{aligned}
20 \lg \left(N_{n}\right)= & -0.5+35 \lg \left(\zeta_{n}\right)+10 \lg \left(F_{s}\right) \\
\zeta_{n}= & \frac{((2 \pi \cdot \text { Dis_n }) / \lambda)}{\left(\left(2 \pi \cdot k_{e} \cdot R_{E}\right) / \lambda\right)^{2 / 3}} ; \quad n=1,2,3 \\
20 \lg \left(F_{s}\right)= & -0.048 \zeta_{n}^{3}+1.0875 \zeta_{n}^{2}+4.0782 \zeta_{n} \\
& -0.8806 \\
L_{3}= & 0.0086 \zeta_{3}^{3}+0.2063 \zeta_{3}^{2}+11.0997 \zeta_{3} \\
& -0.8934
\end{aligned}
$$

Here, $F_{s}$ and $L_{3}$ are given by approximated polynomial functions from [6]. After that, the total path loss $L_{e_{-} \text {diff }}$ is represented as follows.

If $d \geq d_{\text {sum }}, L_{3}$ will be treated as a loss because Dis_3 is more than 0 [6]. Thus, $L_{e_{-} \text {diff }}$ is obtained as

$$
L_{e_{-} \text {diff }}=L_{1}+L_{2}-\left|L_{3}\right| \text {. }
$$

If $d<D_{06}$, the diffraction loss $L_{e_{-} \text {diff }}$ will be equal to 0 because the radio link is over the horizon

$$
L_{e_{-} \text {diff }}=0 .
$$

In this case, the earth curvature can be ignored.

If $D_{06} \leq d<d_{\text {sum }}, L_{3}$ will be regarded as a gain owing to the fact that $d$ is shorter than (Dis_1 + Dis_2) [6]. Thus, the $L_{e_{-} \text {diff }}$ can be represented by

$$
L_{e_{-} \text {diff }}=L_{1}+L_{2}+\left|L_{3}\right| \text {. }
$$

Last but not least, limited by the law of conservation of energy, $L_{e_{-} \text {diff }}$ also should be set to zero when $\left|L_{3}\right|$ is bigger than $\left|L_{1}\right|+\left|L_{2}\right|$ in this situation.

Based on the above-mentioned three measurement campaigns, $D_{06}$ are equal to $11.3590 \mathrm{~km}, 11.3590 \mathrm{~km}$, and $8.0229 \mathrm{~km}$, respectively. Therefore, measurement data 1 with Max.distance $11.06 \mathrm{~km}$ and measurement data 3 with Max.distance $7.778 \mathrm{~km}$ fulfill the condition: $d<D_{06}$. However, in measurement 2, some positions are between $D_{06}$ $(11.3590 \mathrm{~km})$ and $d_{\text {sum }}(38.3895 \mathrm{~km})$, which can be found in Figure 23. It means that the diffraction loss derived from earth curvature effect will appear from $11.3590 \mathrm{~km}$ to $15.01 \mathrm{~km}$. 


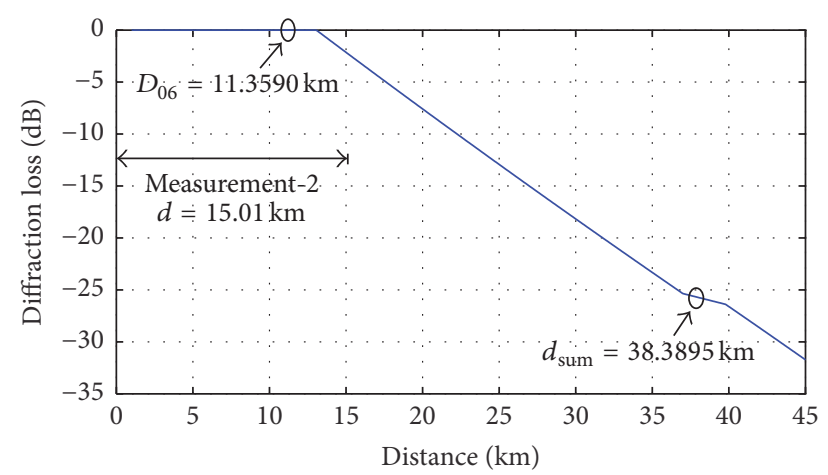

Figure 23: Diffraction loss caused by earth curvature in the case of measurement data_2.
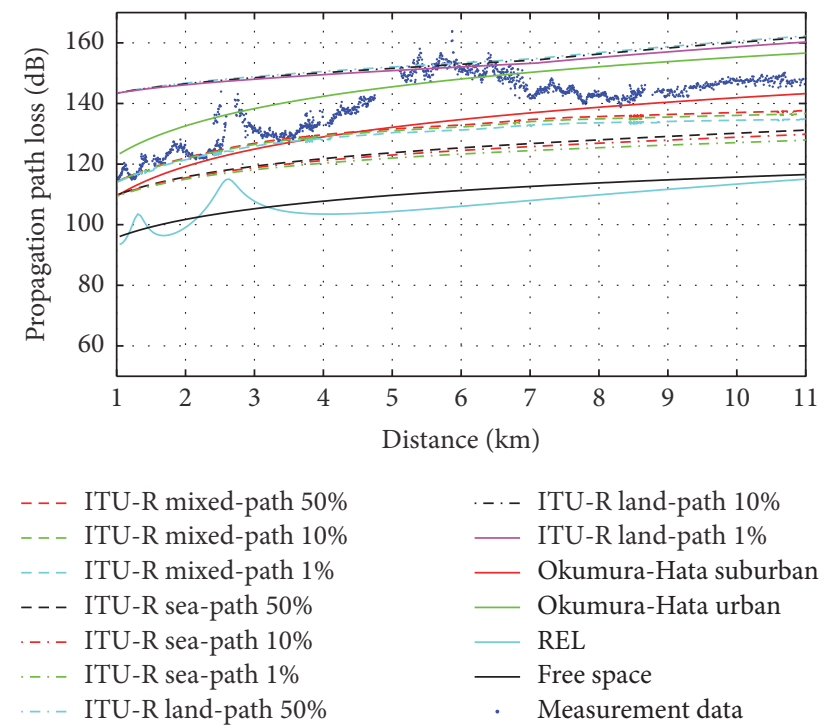

FIGURE 24: The simulation of classic radio propagation path loss models based on measurement data_1.

\section{Parameters Estimation and Simulation Verification}

As mentioned in Section 2, IR model has four empirical parameters $\alpha_{l}, \beta_{l}, \alpha_{s}$, and $\beta_{s}$. Figure 24 shows a series of classic radio propagation path loss models which are widely used in wireless communication such as free space model, REL model, Okumura-Hata model, and ITU-R models. The simulation outcomes indicate that measurement results which are between ITU-R land-path model and sea-path model show obvious mixed-path characteristics. However, ITU-R mixed-path models do not have a good fitting degree, which is displayed in Figure 24. Compared with other models, Okumura-Hata urban model and suburban model match better with the raw data. In addition, both of them are able to be expressed in logarithmic scale $[3,4]$. We assume that the environmental correction factor (shown in Section 2.2 and Figure 3) for all-water regions $M_{s}$ can be depicted by (28):

$$
M_{s}=\alpha_{\text {sub }}-20 \lg \left(\frac{\lambda}{4 \pi}\right)+\left[\beta_{\text {sub }}+2\right] 10 \lg (D),
$$

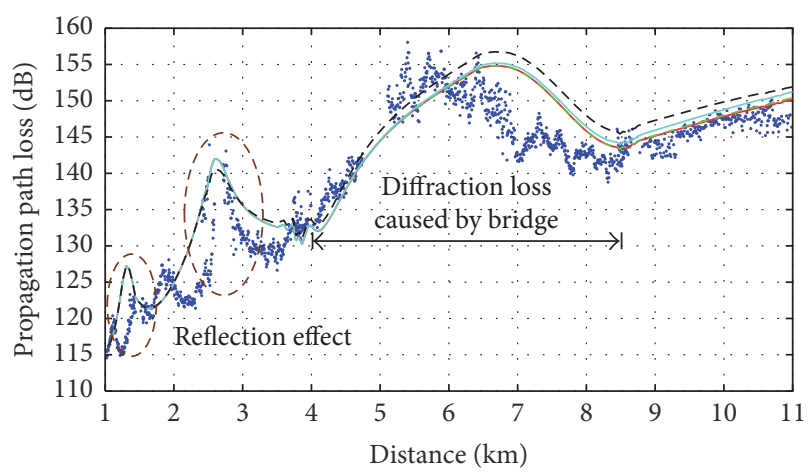

$$
\begin{aligned}
& \text { — IR model } w_{0}^{2}=0.002 \quad--- \text { IR model } w_{0}^{2}=0.02 \\
& - \text { - IR model } w_{0}^{2}=0.004 \\
& \text { IR model } w_{0}^{2}=0.008
\end{aligned}
$$

FIGURE 25: IR model with different $w_{0}^{2}$.

$$
\begin{aligned}
& \alpha_{s}=\alpha_{\text {sub }}-20 \lg \left(\frac{\lambda}{4 \pi}\right), \\
& \beta_{s}=\beta_{\text {sub }}+2 .
\end{aligned}
$$

Here, $\alpha_{\text {sub }}$ and $\beta_{\text {sub }}$ are derived from Okumura-Hata suburban model $[3,4]$. For all-land regions, the OkumuraHata urban model and suburban model are employed to calculate the corresponding correction factors $M_{l}$ (urban or suburban).

In the case of urban environment,

$$
\begin{aligned}
& M_{l}=\alpha_{\mathrm{urb}}-20 \lg \left(\frac{\lambda}{4 \pi}\right)+\left[\beta_{\mathrm{urb}}+2\right] 10 \lg (D) ; \\
& \alpha_{l}=\alpha_{\mathrm{urb}}-20 \lg \left(\frac{\lambda}{4 \pi}\right) \\
& \beta_{l}=\beta_{\mathrm{urb}}+2 .
\end{aligned}
$$

In the case of suburban environment,

$$
M_{l}=M_{s}
$$

Here, $\alpha_{\text {urb }}$ and $\beta_{\text {urb }}$ can be acquired from the OkumuraHata model for urban scenario $[3,4]$. After that, the IR model with different standard deviation of surface height distribution $\sigma_{h}$ and mean-square surface slope $w_{0}^{2}$ (the values of other variables are the same) are described in Figures 25 and 26, respectively. It can be found that the smaller $w_{0}^{2}$ and $\sigma_{h}$ will lead to the higher loss peaks derived from reflection effect. On the basis of the comparison outcomes, IR model chooses 0.002 and 0.2 as the values of $w_{0}^{2}$ and $\sigma_{h}$, which also confirms that the surface of river is smoother than open-sea $\left(w_{0}^{2}=0.25, \sigma_{h}=0.008\right.$ in [6]). Moreover, a good matching degree of diffraction loss proves that the finite dimensions bridge diffraction in IR model are applicable for this scenario.

To verify the universal applicability of IR model, measurement data_2 and measurement data_3 are employed as reference sequences. Figure 27 shows that IR model agrees 


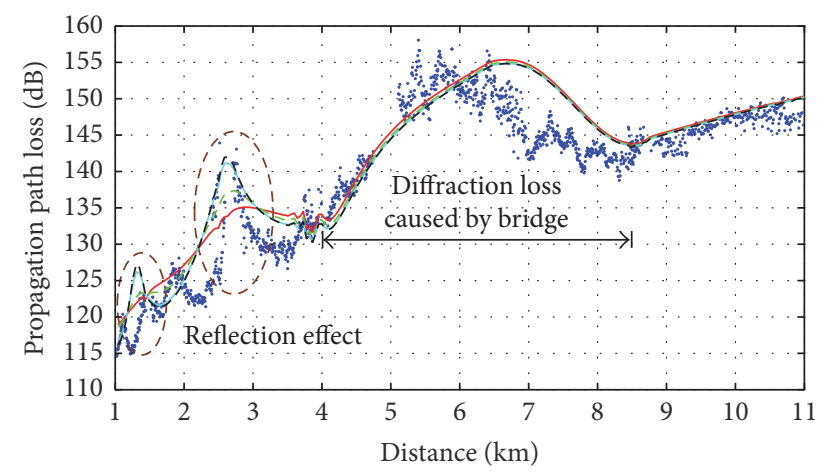

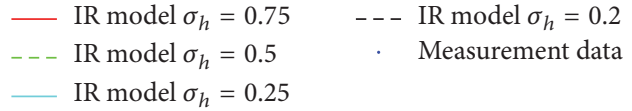

FIGURE 26: IR model with different $\sigma_{h}$.

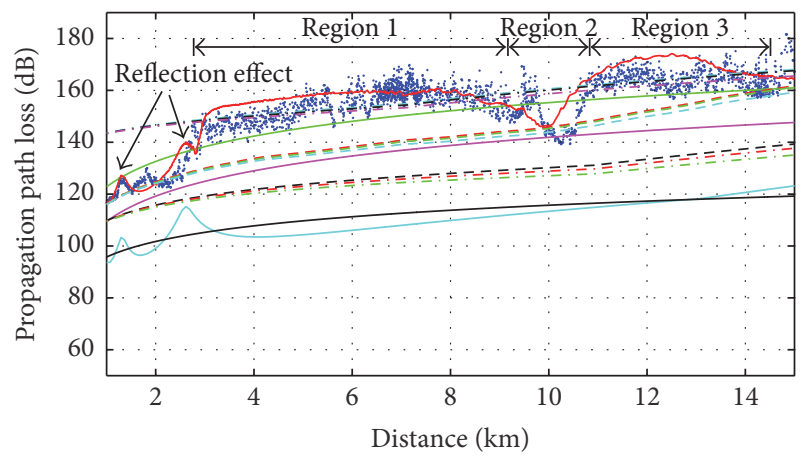

\footnotetext{
- - ITU-R mixed-path $50 \%$

- - - ITU-R mixed-path $10 \%$

- - - ITU-R mixed-path $1 \%$

- - - ITU-R sea-path $50 \%$

-. - ITU-R seat-path $10 \%$

-. - ITU-R sea-path $1 \%$

ITU-R land-path 50\%

-. - ITU-R land-path $10 \%$
}

FIGURE 27: Simulation verification based on measurement data_2. Region 1 denotes the diffraction loss caused by obstacle 1 . The dip in Region 2 is derived from space between the two buildings. Region 3 represents diffraction loss caused by obstacle 2 .

well with the reflection phenomenon and the diffraction loss caused by huge buildings near the river which have great effect on signal transmission. In addition, as mentioned in Section 4.1, the dip occurring around the $10 \mathrm{~km}$ is due to a $40.1 \mathrm{~m}$ space between the two buildings. In Figure 28, both the Okumura-Hata (suburban) model and the IR model match well with the measurement data. In conclusion, these comparisons for the three scenarios prove that the inland river scenario can be approximated as a mixed-path TwoRay geometric model. Secondly, the assumptions of parameters optimization in Section 2 and parameters estimation in Section 5 are reasonable. Thirdly, the finite dimensions method is verified to be an effective way to calculate the diffraction loss caused by bridges, huge buildings, and some other facilities near the river. As shown in Figures 25, 26,

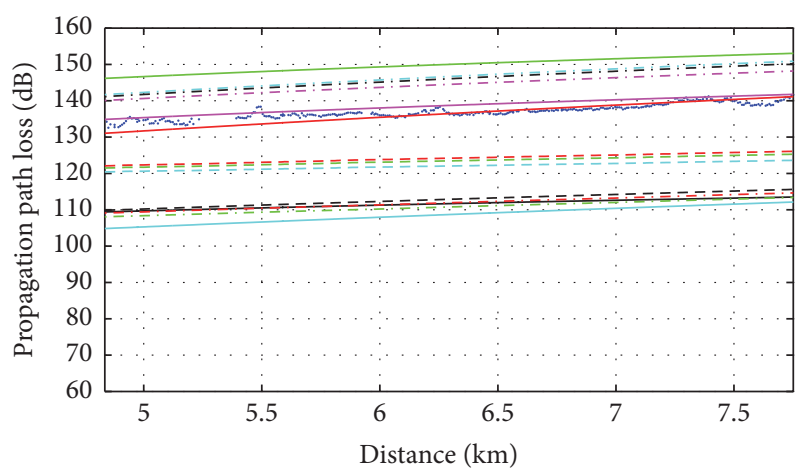

$$
\begin{aligned}
& \text { - - - ITU-R mixed-path 50\% } \\
& \text { - - - ITU-R mixed-path } 10 \% \\
& \text { - - ITU-R mixed-path } 1 \% \\
& \text { - - - ITU-R sea-path 50\% } \\
& \text {...- ITU-R sea-path } 10 \% \\
& \text {-. - ITU-R sea-path } 1 \% \\
& \text { ITU-R land-path 50\% } \\
& \text {... ITU-R land-path 10\% }
\end{aligned}
$$

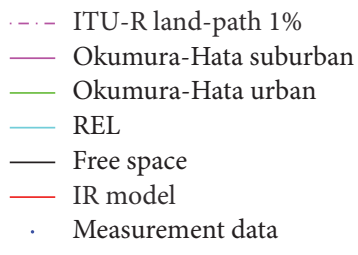

FIGURE 28: Simulation verification based on measurement data_3.

and 27, reflection phenomenon is the dominant influence factor from $1 \mathrm{~km}$ to $3 \mathrm{~km}$, which will bring a loss within the scope of $[0,15] \mathrm{dB}$. On the basis of quantitative analysis in Section 3, diffraction phenomenon is more obvious than other phenomena in $4 \mathrm{~km} \sim 6 \mathrm{~km}$ of measurement 1 and $3 \mathrm{~km} \sim 15 \mathrm{~km}$ of measurement 2 .

\section{Performance Evaluation}

In order to quantify the practicability of these models, three kinds of model selection algorithms are employed. RMSE criteria is a commonly used methodology to evaluate the difference between the theoretical model and measurement data $[11,23,24]$ due to low complexity. By defining the model selection as an "track association problem" in automation and traffic field, [10] provides two new methods (GRG-MAPE and PCC-MAPE) based on Uncertainty-Mathematical Theory, which have been proven more accurate than RMSE.

On the basis of the three methodologies mentioned above, Tables 2, 3, and 4 list the performance evaluation results of path loss models, respectively.

In Table 2, RMSE values denote the error levels of the estimation sequences. Thus, both free space model and REL model are not appropriate for all of these three scenarios due to high RMSE values. As shown in Data_1 and Data_2, Okumura-Hata (urban) model shows smaller RMSE values (7.8181 and 8.0515) than the suburban model and the ITU$\mathrm{R}$ models apart from the IR model (4.6159 and 6.3594). For suburban watercourse scenario (Data_3), IR model and Okumura-Hata (suburban) model are the optimal model and the suboptimal model, respectively. The values in Tables 3 and 4 describe the matching degrees of corresponding path loss models. Afterwards, all of comparison results indicate that IR model performs most effectively for these environments, whereas the Okumura-Hata (suburban) model also matches well with our measurement data in suburban scenario. 
TABLE 2: The RMSE model selection results.

\begin{tabular}{lccc}
\hline \multirow{2}{*}{ Models } & \multicolumn{3}{c}{ Measurements } \\
& Data_1 $^{*}$ & Data_2 $^{*}$ & Data_3* \\
\hline ITU-R land-path 1\% & 13.7119 & 9.3384 & 7.5479 \\
ITU-R land-path 10\% & 14.3341 & 9.3848 & 9.1265 \\
ITU-R land-path 50\% & 14.6268 & 9.4386 & 9.7505 \\
ITU-R sea-path 1\% & 21.0225 & 30.3053 & 26.2342 \\
ITU-R sea-path 10\% & 19.6629 & 28.7838 & 25.0719 \\
ITU-R sea-path 50\% & 18.7016 & 27.7185 & 24.146 \\
ITU-R mixed-path 1\% & 14.4477 & 15.1869 & 15.0571 \\
ITU-R mixed-path 10\% & 13.2697 & 14.1991 & 13.6386 \\
ITU-R mixed-path 50\% & 12.5827 & 13.7837 & 12.9074 \\
Free space model & 30.4055 & 42.5521 & 25.4202 \\
Okumura-Hata (suburban) & 9.2825 & 18.8068 & 1.8525 \\
Okumura-Hata (urban) & 7.8181 & 8.0515 & 12.9709 \\
REL model & 33.3084 & 44.2648 & 28.2632 \\
IR model & 4.6159 & 6.3594 & 1.8310 \\
\hline
\end{tabular}

${ }^{*}$ The smaller RMSE value means better matching degree.

TABLE 3: The GRG-MAPE model selection results.

\begin{tabular}{lccc}
\hline \multirow{2}{*}{ Models } & \multicolumn{3}{c}{ Measurements } \\
& Data_1 $^{*}$ & Data_2 & Data_3* \\
\hline ITU-R land-path 1\% & 0.8833 & 0.9315 & 0.9264 \\
ITU-R land-path 10\% & 0.8795 & 0.9318 & 0.9138 \\
ITU-R land-path 50\% & 0.8774 & 0.9317 & 0.9094 \\
ITU-R sea-path 1\% & 0.8648 & 0.8090 & 0.8064 \\
ITU-R sea-path 10\% & 0.8725 & 0.8175 & 0.8137 \\
ITU-R sea-path 50\% & 0.8779 & 0.8235 & 0.8193 \\
ITU-R mixed-path 1\% & 0.9136 & 0.8976 & 0.8778 \\
ITU-R mixed-path 10\% & 0.9201 & 0.9030 & 0.8892 \\
ITU-R mixed-path 50\% & 0.9240 & 0.9054 & 0.8944 \\
Free space model & 0.7865 & 0.7360 & 0.8123 \\
Okumura-Hata (suburban) & 0.9296 & 0.8774 & 0.9570 \\
Okumura-Hata (urban) & 0.9303 & 0.9378 & 0.8934 \\
REL model & 0.7659 & 0.7206 & 0.7853 \\
IR model & 0.9574 & 0.9522 & 0.9582 \\
\hline
\end{tabular}

${ }^{*}$ The larger GRG-MAPE value means better matching degree.

For the computational complexity of these path loss models, free space model [1], Okumura-Hata suburban model, and its urban model $[3,4]$ are much simpler than others due to lack of sufficient effect factors analysis. ITU-R models [7] own many corrections to the prediction results, including terrain clearance angle correction, antenna height, mixedpath, and tropospheric scattering. However, these commonly used classic empirical models do not analyze deterministic information which is calculated in IR model such as diffraction loss caused by bridges or buildings. Moreover, IR model has three improvements compared with original REL model [6], which can be found in Section 2. Consequently, IR model is more complex than ITU-R models and REL model.
TABLE 4: The PCC-MAPE model selection results.

\begin{tabular}{lccc}
\hline \multirow{2}{*}{ Models } & \multicolumn{3}{c}{ Measurements } \\
& Data_1 $^{*}$ & Data_2 $^{*}$ & Data_3* \\
\hline ITU-R land-path 1\% & 0.8891 & 0.9321 & 0.9419 \\
ITU-R land-path 10\% & 0.8848 & 0.9324 & 0.9317 \\
ITU-R land-path 50\% & 0.8832 & 0.9329 & 0.9276 \\
ITU-R sea-path 1\% & 0.8773 & 0.8165 & 0.8195 \\
ITU-R sea-path 10\% & 0.8838 & 0.8246 & 0.8271 \\
ITU-R sea-path 50\% & 0.8884 & 0.8305 & 0.8329 \\
ITU-R mixed-path 1\% & 0.9263 & 0.9046 & 0.8932 \\
ITU-R mixed-path 10\% & 0.9318 & 0.9102 & 0.9024 \\
ITU-R mixed-path 50\% & 0.9352 & 0.9127 & 0.9070 \\
Free space model & 0.7967 & 0.7447 & 0.8242 \\
Okumura-Hata (suburban) & 0.9390 & 0.8851 & 0.9797 \\
Okumura-Hata (urban) & 0.9398 & 0.9458 & 0.9058 \\
REL model & 0.7581 & 0.7173 & 0.8058 \\
IR model & 0.9688 & 0.9612 & 0.9822 \\
\hline
\end{tabular}

${ }^{*}$ The larger PCC-MAPE value means better matching degree.

\section{Conclusion}

In this paper, a new propagation path loss model based on the REL model is proposed for inland river environment. Compared with the REL model, this model shows three improvements derived from differences between open-sea and inland river. In the aspect of parameters optimization, the formula in $\mathrm{dB}$ scale transformed from the free space model is replaced by the Okumura-Hata model. Secondly, an additional diffraction loss caused by the obstacles near the river has been included. Then, the mixed-path feature is taken into consideration by using the methodology from Recommendation ITU-R P.1546-5. To acquire reference groups and perform parameters estimation, three measurement campaigns have been conducted for different scenarios. According to effect factors analysis and simulation results, the high matching degree of IR model indicates that the radio channel of inland river scenario can be simplified by using Two-Ray geometric model and the method of linear parameters optimization as well as the calculation of finite dimensions diffraction loss are effective. Besides, the comparison between the measurement results and classical path loss models suggests that propagation path without mixture of land and water path is not appropriate for inland river owing to its special mixed-path characteristics. In order to better evaluate the model's effectiveness, RMSE, GRGMAPE, and PCC-MAPE are used for model selection. The corresponding outcomes also prove that our IR model which considers mixed path and some deterministic information matches best with the measurement results.

\section{Competing Interests}

The authors declare that there is no conflict of interests regarding the publication of this paper. 


\section{Acknowledgments}

The authors would like to express their sincere thanks to the ZTE for their generous equipment support, Super Radio AS for technical support on data analysis, and Changjiang Maritime Safety Administration for their coordination work during the measurement process. The work of Kun Yang was supported by the MAMIME project under Grant Agreement no. 256309 by Norwegian Research Council.

\section{References}

[1] A. F. Molisch, Wireless Communications, John Wiley \& Sons, New York, NY, USA, 2007.

[2] J. D. Parsons, The Mobile Radio Propagation Channel, John Wiley \& Sons, New York, NY, USA, 2000.

[3] Y. Okumura, E. Ohmori, and T. Kawano, "Field strength and its variability in VHF and UHF land-mobile radio service," Review of the Electrical Communication Laboratory, vol. 16, no. 9, pp. 825-873, 1968.

[4] M. Hata, "Empirical formula for propagation loss in land mobile radio services," IEEE Transactions on Vehicular Technology, vol. 29, no. 3, pp. 317-325, 1980.

[5] L. Klozar and J. Prokopec, "Propagation path loss models for mobile communication," in Proceedings of the 21st International Conference, Radioelektronika, pp. 287-290, IEEE, Brno, Czech Republic, April 2011.

[6] K. Yang, A. F. Molisch, T. Ekman, and T. Røste, "A deterministic round earth loss model for open-sea radio propagation," in Proceedings of the IEEE 77th Vehicular Technology Conference (VTC Spring '13), pp. 1-5, IEEE, Dresden, Germany, June 2013.

[7] Recommendation ITU-R P.1546-5, "Method for point-toarea predictions for terrestrial services in the frequency range $30 \mathrm{MHz}$ to $3000 \mathrm{MHz}$," September 2013.

[8] N. H. Lu, "Linearized, unified two-ray formulation for propagation over a plane earth," in Proceedings of the Sensors for Industry Conference, pp. 95-100, IEEE, Houston, Tex, USA, February 2005.

[9] M. R. Akdeniz, Y. Liu, M. K. Samimi et al., "Millimeter wave channel modeling and cellular capacity evaluation," IEEE Journal on Selected Areas in Communications, vol. 32, no. 6, pp. 11641179, 2014.

[10] J. Yu, C. Li, K. Yang, and W. Chen, "GRG-MAPE and PCCMAPE based on uncertainty-mathematical theory for pathloss model selection," in Proceedings of the 83rd IEEE Vehicular Technology Conference (VTC Spring '16), May 2016.

[11] C. Li, J. Yu, K. Yang, and W. Chen, "Grey System Theory application in model selection of channel modeling at $1.4 \mathrm{GHz}$," in Proceedings of the International Conference on Wireless Communications, Signal Processing and Networking (WiSPNET '16), pp. 1745-1749, IEEE, Chennai, India, March 2016.

[12] 3GPP, "Physical channels and modulation," 3GPP TS 36.211, Technical Specification Group Radio Access Network.

[13] J. Epstein and D. W. Peterson, "An experimental study of wave propagation at $850 \mathrm{MC}$," Proceedings of the IRE, vol. 41, no. 5, pp. 595-611, 1953.

[14] J. Deygout, "Multiple knife-edge diffraction of microwaves," IEEE Transactions on Antennas and Propagation, vol. 14, no. 4, pp. $480-489,1966$.

[15] Recommendation ITU-R P.526-13, "Propagation by diffraction," November 2013.
[16] E. Torabi, A. Ghorbani, and A. Tajvidy, "A modified diffraction coefficient for imperfect conducting wedges and buildings with finite dimensions," IEEE Transactions on Antennas and Propagation, vol. 57, no. 4, pp. 1197-1207, 2009.

[17] M. B. Raza and T. Fickenscher, "Diffraction loss and phase modulation of terrestrial radio-link by wind turbine," in Proceedings of the International Workshop on Antenna Technology: Small Antennas, Novel EM Structures and Materials, and Applications (iWAT '14), pp. 382-384, Sydney, Australia, March 2014.

[18] K. Yang, T. Ekman, T. Roste et al., "A quasi-deterministic path loss propagationmodelfortheopenseaenvironment," in Proceedings of the IEEE 14th International Symposium on Wireless Personal Multimedia Communications (WPMC '11), pp. 1-5, October 2011.

[19] K. Yang, Channel measurements and channel modeling for the open sea [Ph.D. thesis], Norwegian University of Science and Technology (NTNU), Trondheim, Norway, 2013.

[20] B. Smith, "Geometrical shadowing of a random rough surface," IEEE Transactions on Antennas and Propagation, vol. 15, no. 5, pp. 668-671, 1967.

[21] K. Bullington, "Radio propagation at frequencies above 30 megacycles," Proceedings of the IRE, vol. 35, no. 10, pp. 1122-1136, 1947.

[22] K. Yang, T. Roste, F. Bekkadal, K. Husby, and O. Trandem, "Long-distance propagation measurements of mobile radio channel over sea at $2 \mathrm{GHz}$," in Proceedings of the IEEE Vehicular Technology Conference (VTC Fall '11), September 2011.

[23] M. Farhoud, A. El-Keyi, and A. Sultan, "Empirical correction of the Okumura-Hata model for the $900 \mathrm{MHz}$ band in Egypt," in Proceedings of the 3rd International Conference on Communications and Information Technology (ICCIT '13), pp. 386-390, IEEE, June 2013.

[24] I. Rodriguez, H. C. Nguyen, T. B. Sorensen et al., "A geometricalbased vertical gain correction for signal strength prediction of downtilted base station antennas in urban areas," in Proceedings of the 76th IEEE Vehicular Technology Conference (VTC '12), pp. 1-5, IEEE, Quebec, Canada, September 2012. 


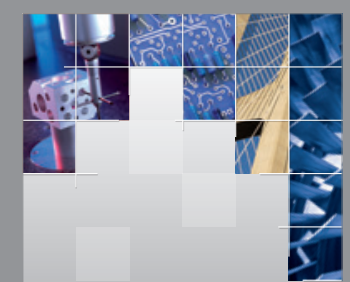

\section{Enfincering}
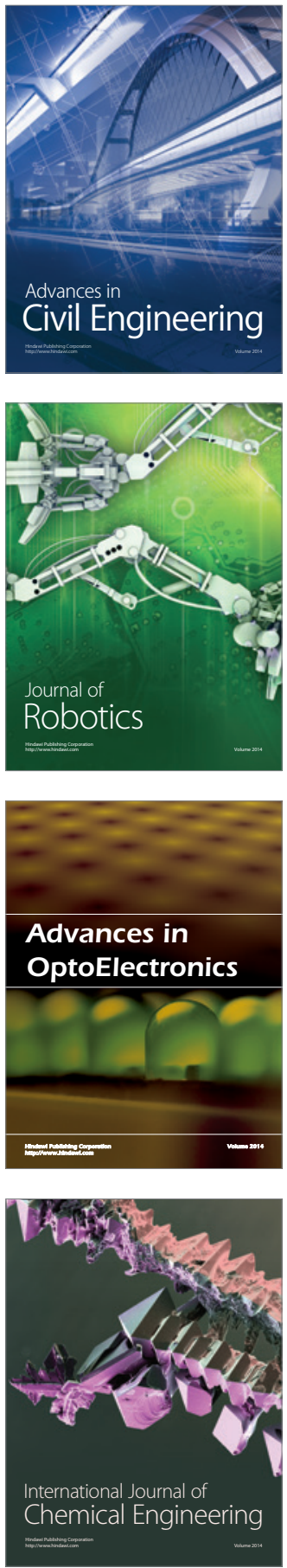

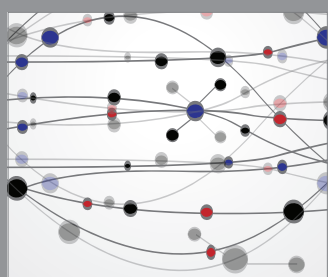

The Scientific World Journal

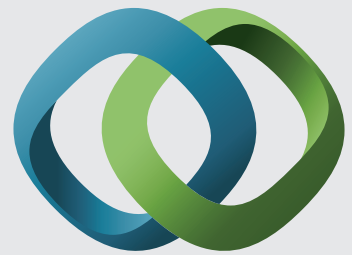

\section{Hindawi}

Submit your manuscripts at

https://www.hindawi.com
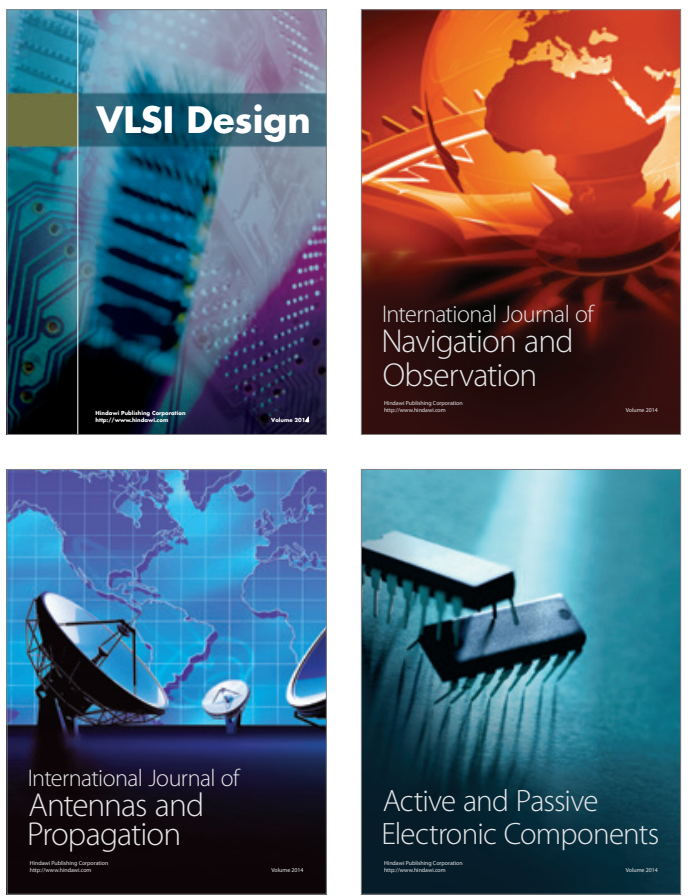
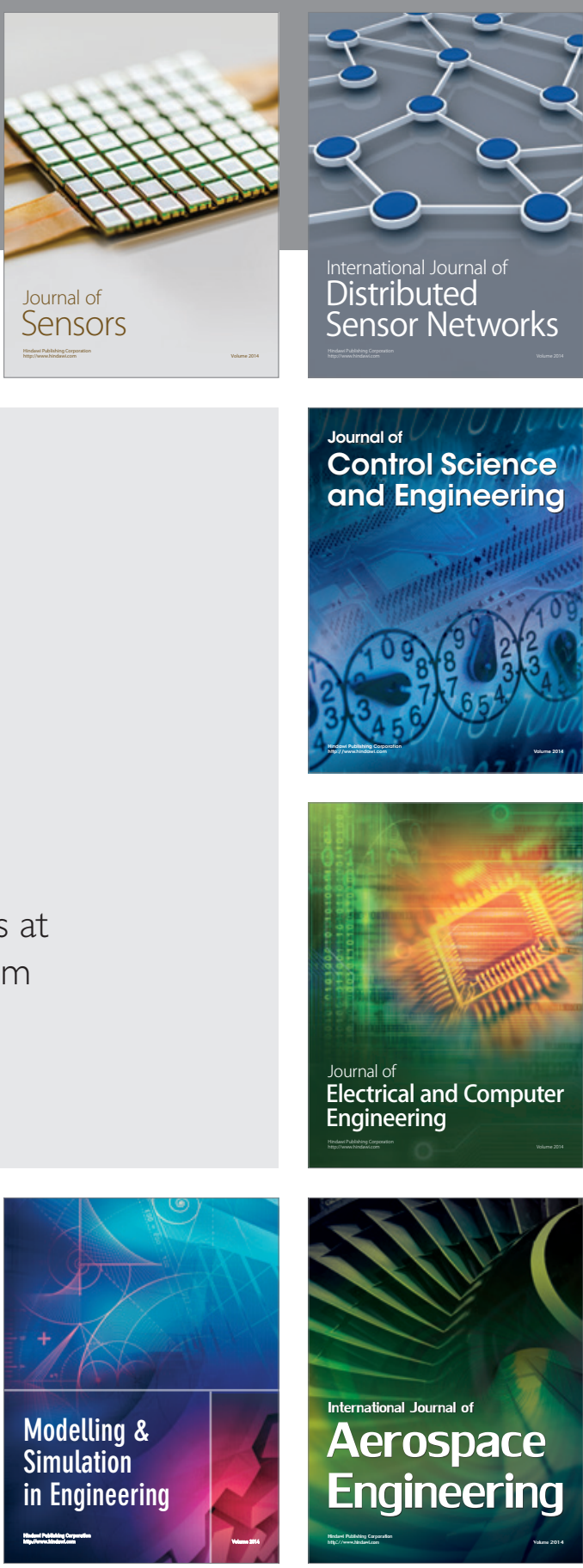

International Journal of

Distributed

Sensor Networks

$-$

Joumal of

Control Science

and Engineering
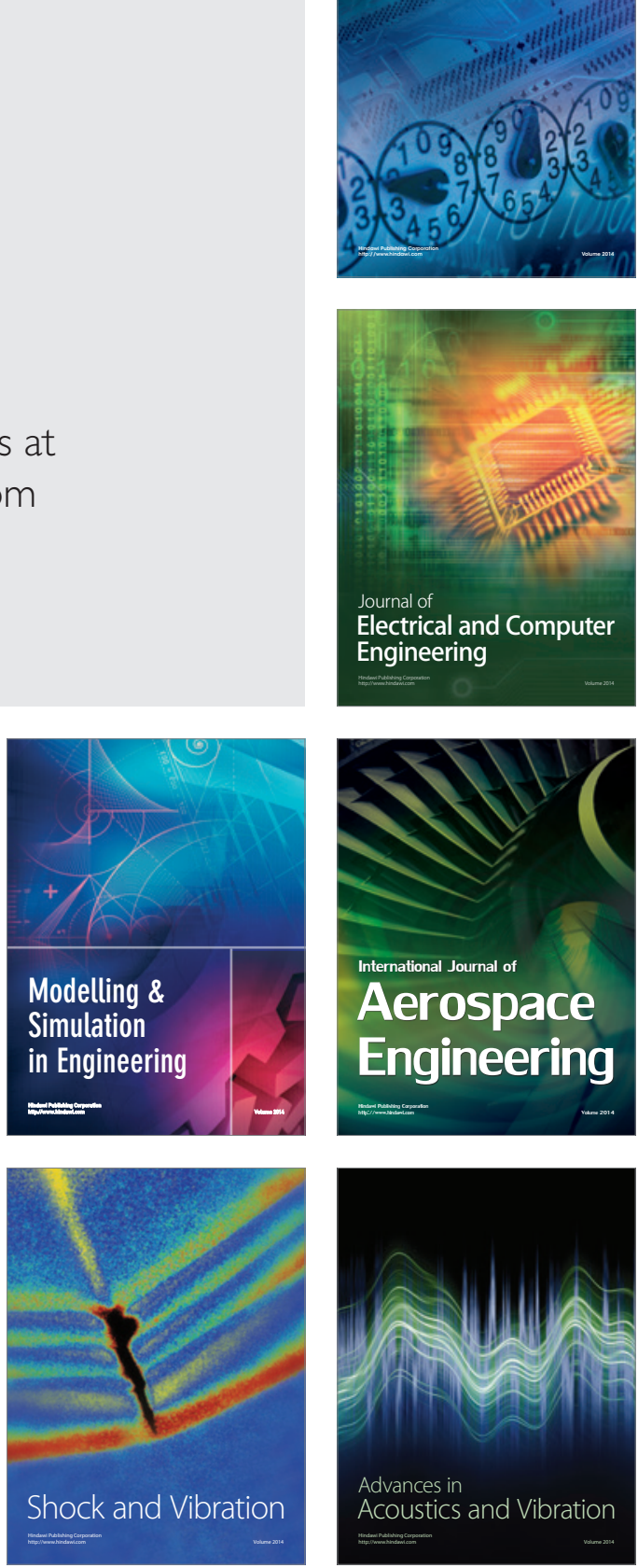